\title{
Biomaterial vaccines capturing pathogen- associated molecular patterns protect against bacterial infections and septic shock
}

\author{
Michael Super ${ }^{1,2,4}$, Edward J. Doherty ${ }^{1,4}$, Mark J. Cartwright ${ }^{1}$, Benjamin T. Seiler ${ }^{1}$ Fernanda Langellotto', \\ Nikolaos Dimitrakakis', Des A. White', Alexander G. Stafford', Mohan Karkada', Amanda R. Graveline', \\ Caitlin L. Horgan', Kayla R. Lightbown', Frank R. Urena ${ }^{1}{ }^{1}$, Chyenne D. Yeager', Sami A. Rifai', \\ Maxence O. Dellacherie ${ }^{3}$, Aileen W. Li ${ }^{3}$, Collin Leese-Thompson', Hamza ljaz', Amanda R. Jiang ${ }^{2}$, \\ Vasanth Chandrasekhar', Justin M. Scott ${ }^{10}{ }^{1}$, Shanda L. Lightbown', Donald E. Ingber ${ }^{12,2,3}$ and \\ David J. Mooney ${ }^{1,3 凶}$
}

Most bacterial vaccines work for a subset of bacterial strains or require the modification of the antigen or isolation of the pathogen before vaccine development. Here we report injectable biomaterial vaccines that trigger potent humoral and T-cell responses to bacterial antigens by recruiting, reprogramming and releasing dendritic cells. The vaccines are assembled from regulatorily approved products and consist of a scaffold with absorbed granulocyte-macrophage colony-stimulating factor and CpG-rich oligonucleotides incorporating superparamagnetic microbeads coated with the broad-spectrum opsonin Fc-mannose-binding lectin for the magnetic capture of pathogen-associated molecular patterns from inactivated bacterial-cell-wall lysates. The vaccines protect mice against skin infection with methicillin-resistant Staphylococcus aureus, mice and pigs against septic shock from a lethal Escherichia coli challenge and, when loaded with pathogen-associated molecular patterns isolated from infected animals, uninfected animals against a challenge with different $E$. coli serotypes. The strong immunogenicity and low incidence of adverse events, a modular manufacturing process, and the use of components compatible with current good manufacturing practice could make this vaccine technology suitable for responding to bacterial pandemics and biothreats.

D eaths from drug-resistant microbial infections are increasing $^{1,2}$, approvals of new antibiotic classes are falling ${ }^{1,2}$, and there are increasing concerns about engineered biothreat agents that may be difficult to treat with conventional therapeutics $^{3}$. Although broad-spectrum infection vaccines could be used to confront the challenges related to increases in drug-resistant bacterial infections and decreases in approvals of new antibiotics, the major antigens on bacterial-cell-wall surfaces are poorly immunogenic serotype-specific polysaccharides that do not easily support vaccine production ${ }^{4,5}$. Some bacterial vaccines have been developed using live-attenuated bacteria (for example, BCG for Mycobacterium tuberculosis) but these have outgrowth risks, especially in immunosuppressed patients ${ }^{6}$. Other vaccines use inactivated bacteria or inactivated toxins ${ }^{7}$, but given that purified carbohydrate vaccines are poorly immunogenic and require multiple boosts ${ }^{8}$, carbohydrate antigens are often chemically conjugated to proteins to increase their efficacy ${ }^{9}$; however, these approaches are limited to only a subset of bacterial strains ${ }^{10}$ or require the modification of the antigen ${ }^{11}$. Moreover, all existing vaccine modalities require that the pathogen or antigens be isolated before a vaccine can be developed, which may not be possible with rapidly spreading pathogens or engineered biothreat agents. Importantly, there are no approved vaccines for the most common pathogens causing sepsis, including Gram-negative Escherichia coli, Gram-positive
Staphylococcus aureus or the methicillin-resistant form of S. aureus (MRSA).

We aimed to develop a broad-spectrum infection vaccine that is potent, durable and safe, using complex killed bacterial components that do not require cold-chain storage and can be used for ring vaccination in biothreat or pandemic emergencies, without having to first identify or isolate the pathogen. To address this challenge, we combined two technologies that are currently in clinical development for other applications-a broad-spectrum engineered opsonin known as Fc-mannose-binding lectin (FcMBL $)^{12-14}$ that rapidly binds to carbohydrate-containing pathogen-associated molecular pattern (PAMP) antigens (including glycoproteins and glycolipids) expressed on the surface of over 120 different pathogen species and toxins, including some that are known to trigger innate immunity (such as lipopolysaccharide endotoxin) ${ }^{15}$, and a biomaterial scaffold-based vaccine used for cancer immunotherapy ${ }^{16-18}$ - to create a new composite infection vaccine technology, which we named ciVAX (Fig. 1). Nanoparticle-based biomaterial vaccines have been shown to generate humoral and cellular immune responses ${ }^{19}$. However, the size and polydispersity of nanoparticles as well as their shape, surface chemistry, physical properties and ability to escape from endosomes have all been shown to be key to their ability to target their cargo to antigen-presenting cells and their effectiveness ${ }^{20}$. Here we increase vaccine engagement with dendritic cells

'Wyss Institute for Biologically Inspired Engineering, Harvard University, Boston, MA, USA. ${ }^{2}$ Vascular Biology Program and Department of Surgery, Boston Children's Hospital and Harvard Medical School, Boston, MA, USA. ${ }^{3}$ Harvard John A. Paulson School of Engineering and Applied Sciences, Harvard University, Cambridge, MA, USA. ${ }^{4}$ These authors contributed equally: Michael Super, Edward J. Doherty. $\varpi_{e}$-mail: David.Mooney@wyss.harvard.edu 


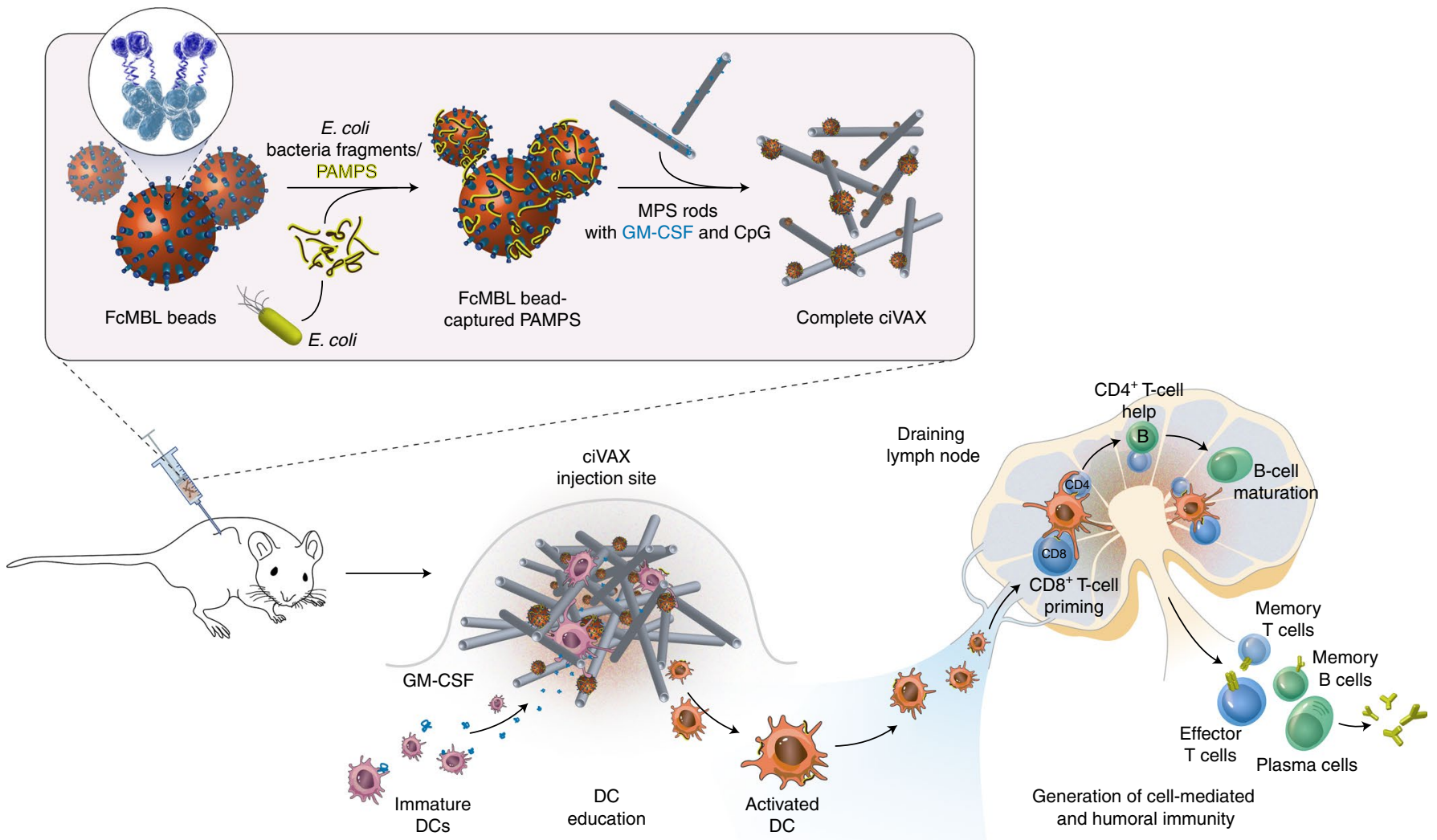

Fig. 1 | Production and application of the ciVAX vaccine. Fragments of killed bacteria (such as E. coli) are exposed to beads coated with FcMBL, leading to the capture of PAMPs on the beads. The beads are then combined with MPS rods with absorbed GM-CSF and CpG-rich oligonucleotides to form the complete ciVAX. Needle injection of ciVAX results in the accumulation of immature dendritic cells within the scaffold formed from the MPS rods owing to the release of GM-CSF and in the subsequent maturation of these cells on exposure to CpG. Activated dendritic cells traffic PAMPs from the ciVAX to the draining lymph node, interact with resident B and T cells, and generate humoral and cell-mediated immune responses to the bacterial antigens. DC, dendritic cell.

using macroscale biomaterials that release granulocyte-macrophage colony-stimulating factor (GM-CSF), as this approach has been shown to substantially increase not only dendritic cells at the vaccination site, but also the numbers of antigen-presenting dendritic cells in the draining lymph nodes. Combining biomaterial-based vaccines with FcMBL capture of antigens provides several advantages, including a widely applicable approach to antigen inclusion in a vaccine, the ability to concentrate and manipulate antigen-presenting cells to enhance adaptive immunity and a plug-and-play nature to the vaccine construction.

To construct ciVAX, bacteria are inactivated using irradiation or antibiotic treatment, the PAMPs are then captured using FcMBL-coated magnetic beads (FcMBL beads), which are mixed with mesoporous silica (MPS) microparticles with adsorbed GM-CSF and synthetic oligonucleotides rich in unmethylated cytosine and guanine nucleotides (CpG). The ciVAX vaccine is then injected subcutaneously, where the MPS particles spontaneously self-assemble into a porous biomaterial matrix, which acts as a release depot for GM-CSF that leads to the accumulation dendritic cells and CpG, which activates these cells ${ }^{16}$ while they process the provided antigen source. The MPS degrades due to hydrolysis and is safely excreted by the kidneys as silicic acid after approximately 27 days $^{21}$. In some experiments we used pre-formed porous scaffolds fabricated from poly(lactide-co-glycolide) (PLG). The technology is modular-to produce ciVAX for a new pathogen, all that needs to be exchanged is the PAMP fractions from the inactivated pathogen. ciVAX can even be used for vaccination using antigens directly captured and killed from infected animals and hence could be used with autogenous biologics for veterinary applications (APHIS, USDA) and in the context of pandemics, zoonotic diseases and unknown biothreat agents.

\section{Results}

ciVAX produces robust humoral and cellular immune responses with minimal adverse events. In our initial experiments we did not use FcMBL to capture PAMPs; instead, we used the whole-cell lysate made from killed antibiotic-resistant $E$. coli RS218 bacteria to test for immunogenicity, adverse events (per Code of Federal Regulations (CFR), an adverse event (AE) is defined as any untoward medical occurrence associated with the use of a drug) and safety in rabbits. In these pilot studies we found that the whole-cell lysate resulted in strong adverse events and the production of necrotic lesions at the injection site (Fig. 2a). To avoid this toxicity, we then captured the E. coli PAMPs from the lysate using FcMBL beads ${ }^{13,14}$. When FcMBL bead-captured PAMPs containing an equivalent amount

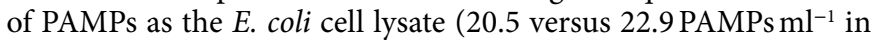
lysate) were injected into rabbits (Fig. 2b), there were no adverse events and there was little evidence of necrosis at the injection site in the FcMBL-bead condition (Fig. 2b). Polyacrylamide electrophoretic analysis of FcMBL-captured PAMPs containing an equivalent amount of lipopolysaccharide endotoxin as the E. coli cell lysate (1,400 and 1,480 EU (endotoxin units), respectively; measured 
a

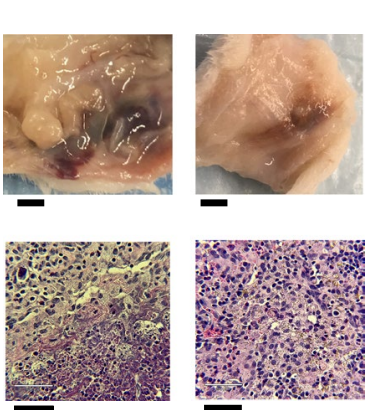

c

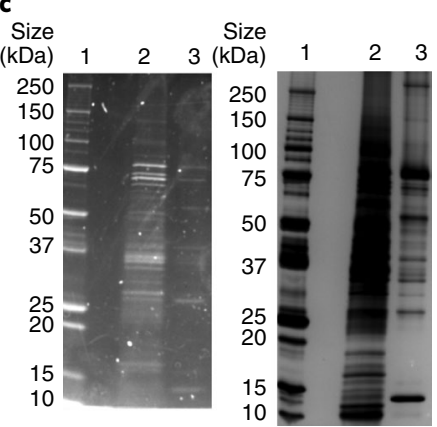

d

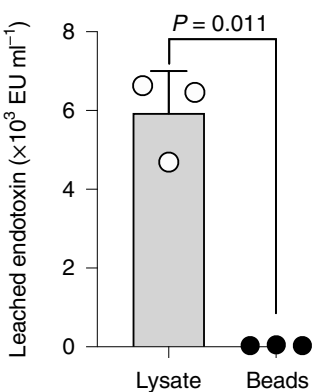

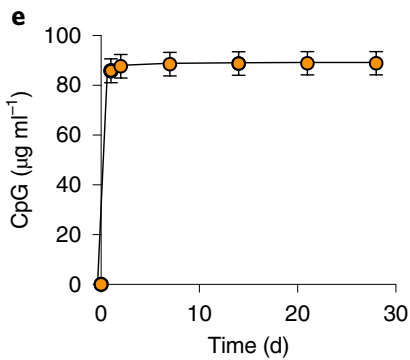
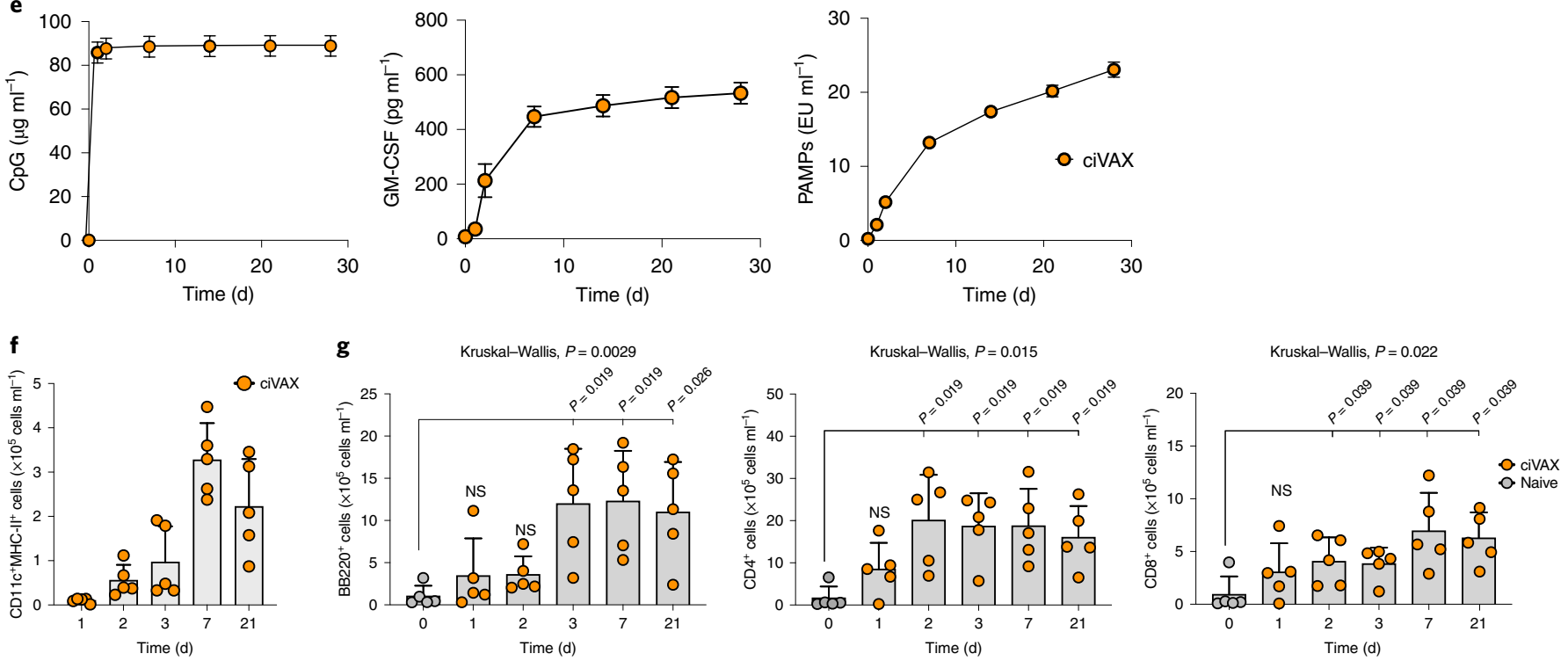

Fig. 2 | ciVAX presents a subset of bacterial-cell-wall PAMPs in a biomaterial scaffold that recruits dendritic cells to the injection site and produces robust humoral and cellular immune responses with minimal adverse events. $\mathbf{a}, \mathbf{b}$, Gross pathology (top) and histology ( $\times 40$; bottom) of the injection site following whole-cell-lysate injection into the flank of rabbits (a) compared with sites of FcMBL-captured PAMPs injection (b). The injection sites were imaged 2 weeks after immunization ( $n=3$; one representative example shown). Scale bars, $1 \mathrm{~cm}$ (top) and $50 \mu \mathrm{m}$ (bottom). c, Coomassie brilliant Fluor (left) and silver staining (right) of an SDS-PAGE gel of molecular weight marker (lane 1) as well as equivalent amounts of endotoxin from the RS218 lysate (1,480 EU; lane 2) and FcMBL bead-captured PAMPs from the RS218 lysate (1,400 c.f.u.; lane 3). d, In vitro study of RS218 endotoxin PAMPs leaching from MPS-scaffold ciVAX without FcMBL beads (lysate) or from intact ciVAX with FcMBL beads $(n=3)$ over 1h. e, Kinetic study of release of CpG, GM-CSF and endotoxin from ciVAX over a 28-day study in vitro $(n=3) . \mathbf{f}, \mathbf{g}$, Numbers of activated dendritic cells over time-as indicated by CD11c and MHC-II stainingat the ciVAX injection site $(\mathbf{f})$ and numbers of $B$ and T cells in the draining lymph node as a function of time post vaccination ( $\mathbf{g} ; n=5$ mice per group). In $\mathbf{d}-\mathbf{g}$, data are the mean \pm s.d. In $\mathbf{d}, \mathbf{g}$, significant differences were identified using an unpaired Student's $t$-test and Mann-Whitney $U$ tests, respectively. NS, not significant.

using an Endosafe LAL assay), followed by Coomassie (Fig. 2c) and silver staining showed that although the FcMBL bead-captured material was enriched for PAMPs, it contained less protein than the lysate. Liquid chromatography with tandem mass spectrometry (LC-MS/MS) analysis was then used to thoroughly characterize the captured material and this analysis showed that most of the individual proteins in the lysate were captured using FcMBL beads; $88 \%$ of the proteins identified in a total lysate of $E$. coli were also identified on the FcMBL bead-captured fraction, whereas $53 \%$ of $S$. aureus proteins were also in the FcMBL bead-captured fraction, which was enriched for membrane-associated proteins (Supplementary Fig. 1). In a head-to-head comparison, the E. coli RS218 lysate was added directly to MPS vaccines with GM-CSF and $\mathrm{CpG}$, and PAMPs from the RS218 lysate were first pre-captured with FcMBL beads, and these were then incorporated into ciVAX. Significantly more endotoxin leached from the lysate vaccines than from ciVAX in a 1-h in vitro study (Fig. 2d). The release of the various agents from the complete ciVAX was then quantified and CpG was found to be released rapidly, whereas GM-CSF and endotoxin were released more slowly over a 28 -day window in vitro (Fig. 2e). However, substantial quantities of the input of $100 \mu \mathrm{g}$ $\mathrm{CpG}, 3 \mu \mathrm{g}$ GM-CSF and 4,900 EU endotoxin remained associated with the ciVAX at the end of this time. We used the previously described sandwich ELISA-like assay ${ }^{13,14}$ to quantify and equalize the total amount of captured PAMPs added to each vaccine in all of the following studies to ensure equivalent PAMP dosing in different experiments and with different bacterial species. In vivo, the ciVAX forms a subcutaneous depot with little sign of necrosis, and the MPS lasts for longer than 7 days and dissolves spontaneously by 37 days (Supplementary Fig. 2).

When we immunized mice with the E. coli PAMP ciVAX vaccine containing $7.5 \mathrm{ng}$ PAMPs, we detected activated dendritic cells at the injection site within 2 days of the immunization; their numbers continued to increase until day 7 and remained elevated until day 

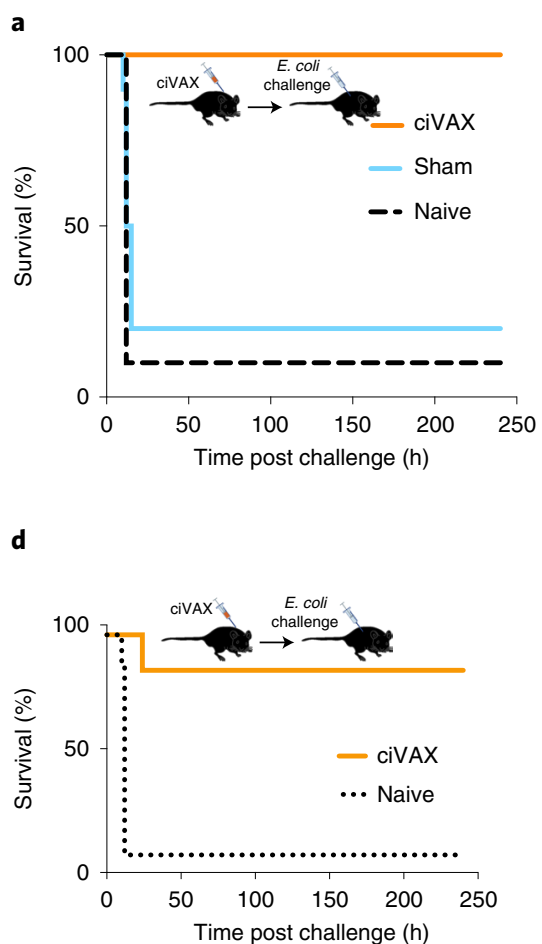

g

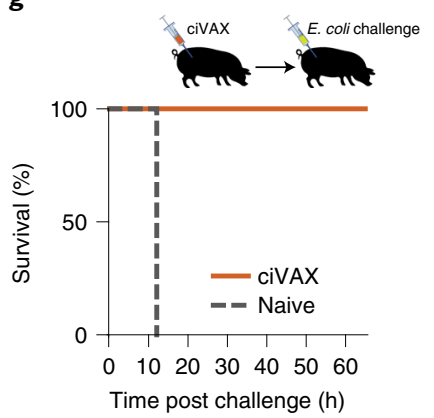

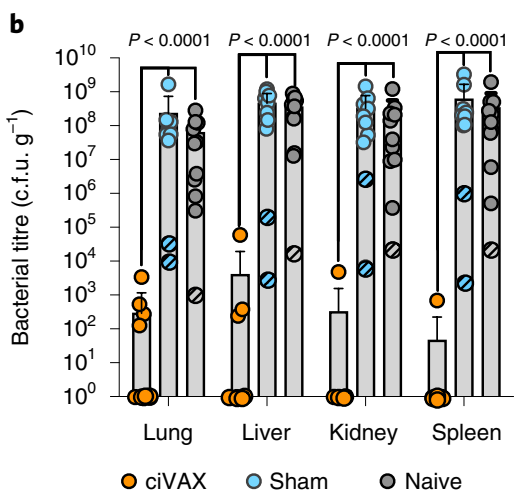

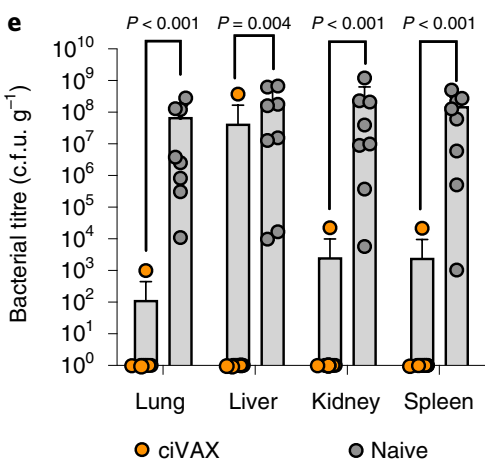

h

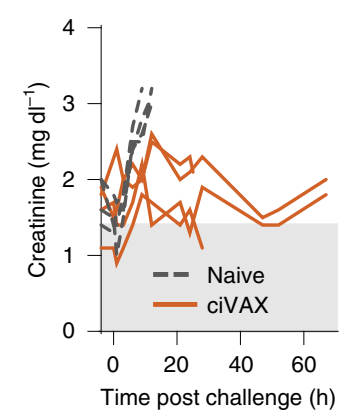

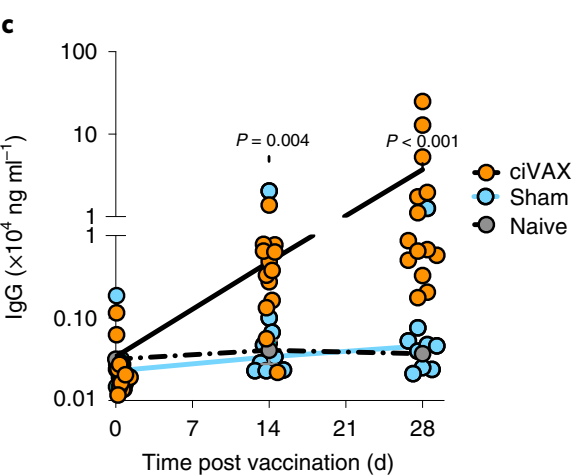

f

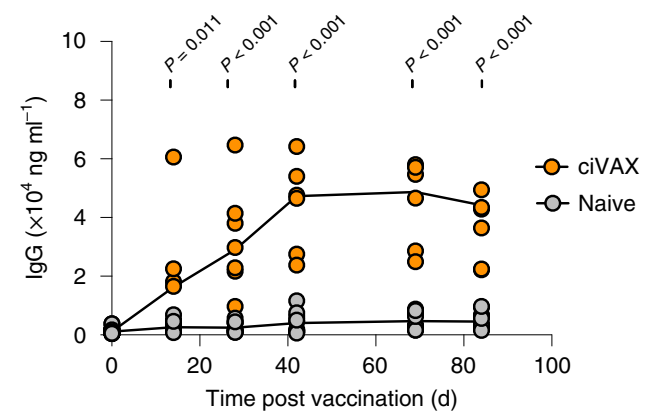

i

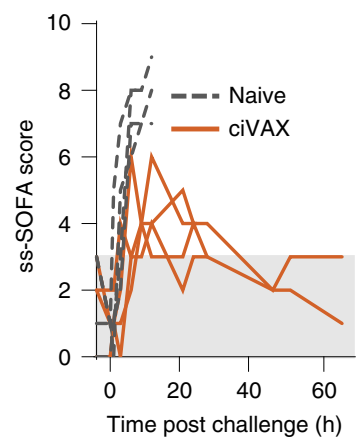

Fig. 3 | ciVAX protects mice from $E$. coli sepsis in an RS218 challenge model and pigs from $E$. coli septic shock. a-f, The ciVAX vaccine was produced by mixing lyophilized MPS biomaterial scaffold containing murine GM-CSF and CpG with cefepime-killed RS218 captured on FcMBL paramagnetic beads. Male and female BALB/c mice (8-10 weeks old) were vaccinated with a single subcutaneous injection of ciVAX or sham scaffold with GM-CSF and CpG but without the FCMBL-RS218, or unvaccinated (naive). The mice were challenged on days 35 (10-12 per group; a-c) or 90 (d-f; eight or nine per group) post vaccination with an intraperitoneal injection of $5 \times 10^{6} \mathrm{c}$.f.u. E. coli (RS218). The mice were monitored for survival for a further $240 \mathrm{~h}$ (10 days). The mice were killed at humane-criteria endpoints or the conclusion of the experiment and their organs were disaggregated using bead milling, followed by quantitative culture. b,e, Bacterial load in the organs isolated from mice in the various experimental groups. The hatched blue and grey symbols depict the sham and naive mice that survived the challenge. $\mathbf{c , f}$, RS218-specific antibodies in mouse serum were measured after vaccination and before challenge, using $\mathrm{F}\left(\mathrm{ab} \mathrm{b}^{\prime}\right)_{2}$ fragment goat anti-mouse lgG. $\mathbf{g}-\mathbf{i}$, The ciVAX for swine was prepared similarly to a-f using the multidrug-resistant clinical isolate $E$. coli 41949 instead of RS218. Female swine (four per group) were vaccinated, boosted on day 28 and challenged 42 days after vaccination with a lethal dose of 41949 delivered by infusion over 4-8 h using the conscious model of sepsis we developed ${ }^{20}$. The Yorkshire pigs (both vaccinated and naive swine) were monitored for survival $(\mathbf{g}$ ), creatinine levels $(\mathbf{h})$ and swine-specific SOFA (ss-SOFA; $\mathbf{i}$ ) over time. Normal levels of creatinine and ss-SOFA are represented by the grey shading. In $\mathbf{b}, \mathbf{e}$, data are the mean \pm s.d. $\ln \mathbf{b}, \mathbf{c}, \mathbf{e}, \mathbf{f}$, statistically significant differences between the independent groups were determined using a non-parametric Mann-Whitney $U$ test.

21 (Fig. 2f). This was accompanied by significantly increased numbers of dendritic, $\mathrm{B}$ and $\mathrm{T}$ cells in the draining lymph nodes (Fig. $2 \mathrm{~g}$ and Supplementary Fig. 3). Past work in cancer with a similar biomaterial-based vaccine demonstrated a similar enrichment of activated dendritic cells at the injection site as well as potent humoral and cellular anti-cancer immune responses ${ }^{16,17,22}$. Immunization of mice with ciVAX lacking GM-CSF and CpG led to a reduction in the total cell number and the number of activated dendritic cells at the injection site as well as a parallel reduction in the total number of cells and the number of activated dendritic, $\mathrm{T}$ and $\mathrm{B}$ cells in the draining lymph node (Supplementary Fig. 3), as expected.

E. coli ciVAX prophylaxis protects mice and pigs. Mice were then vaccinated and challenged with a lethal intraperitoneal injection of the homologous antibiotic-resistant E. coli RS218 strain (O18:H7), isolated from a human neonate with meningitis. Although only $9 \%$ of the unvaccinated mice survived the challenge at 35 days, all of the vaccinated mice remained alive and healthy. Inclusion of 

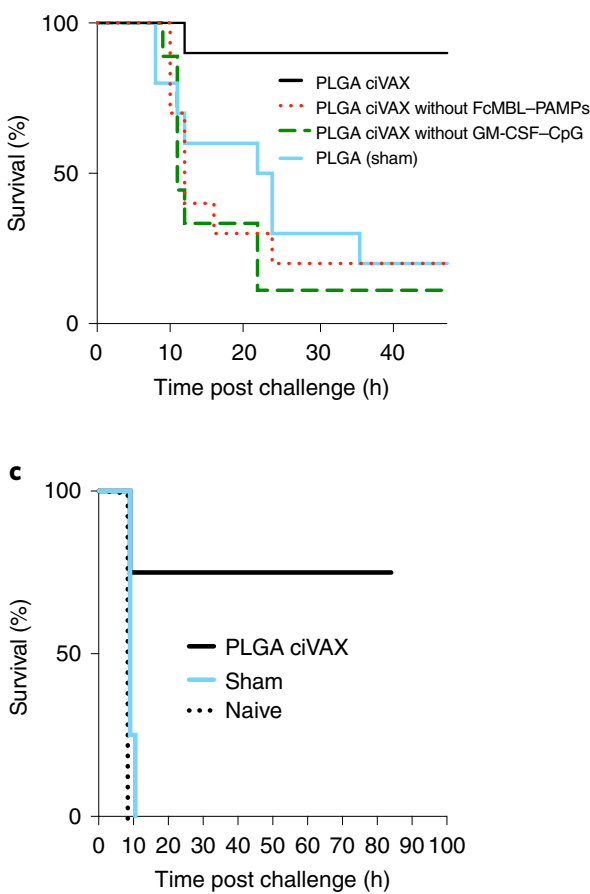

b
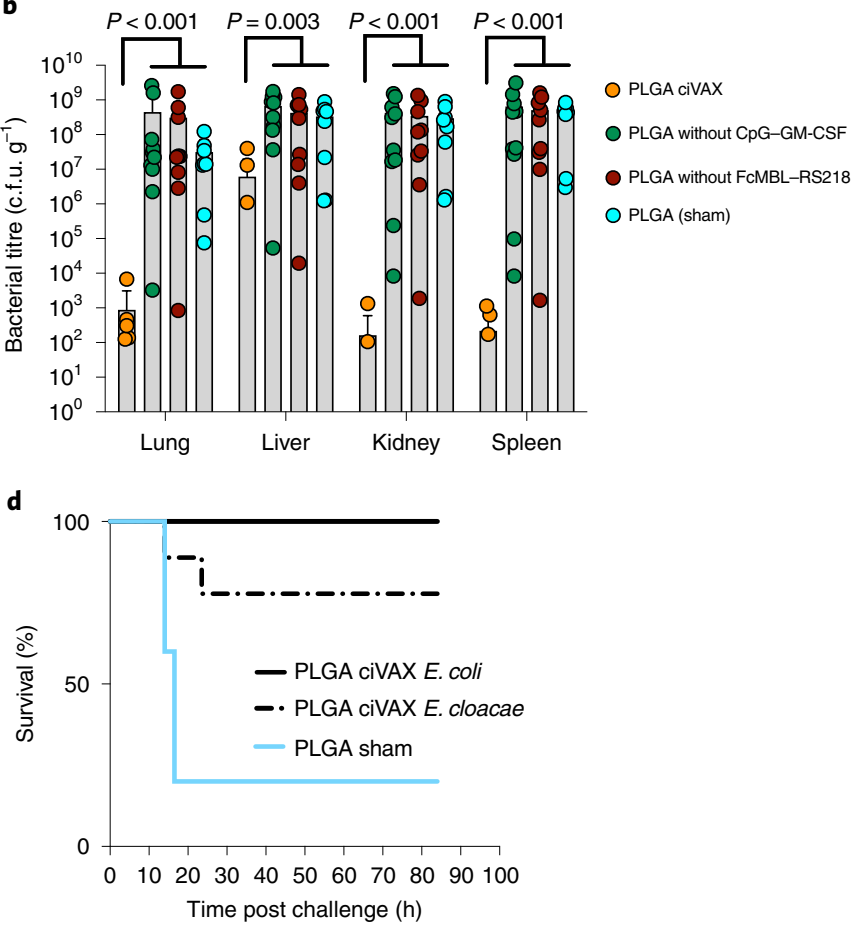

Fig. 4 | The PLG ciVAX vaccine is effective compared with individual components, yields a durable protective immune response and demonstrates cross protection to other Enterobacteriaceae. a-d, PLG ciVAX was produced by mixing PLG acid scaffold containing murine GM-CSF and CpG with cefepime-killed RS218 captured on FcMBL beads, followed by lyophilization. a, Male and female mice (8-10 weeks old) were vaccinated with a single subcutaneous injection of ciVAX, ciVAX without the FcMBL-PAMPs, ciVAX without the GM-CSF-CpG or just PLG sham ( $n=5$ per group). The mice were challenged with an intraperitoneal injection of $5 \times 10^{6}$ c.f.u. E. coli RS218 35 days post vaccination and monitored for mortality and humane criteria for a further $48 \mathrm{~h}$. b. Pathogen load in organs at necropsy for the ciVAX group and the groups without various components of the vaccine ( $n=9$ per group). Data are the mean \pm s.d. Statistically significant differences between multiple independent groups were identified by implementing a non-parametric Kruskal-Wallis test. c, Male and female mice (8-10 weeks old) were vaccinated with a single subcutaneous injection of ciVAX or ciVAX sham without the FcMBL-PAMPs, or left unvaccinated (naive; $n=5$ per group), followed by challenge with $5 \times 10^{6}$ c.f.u. E. coli RS218 on day 90 after vaccination. d, Male and female mice (8-10 weeks old) were vaccinated with a single subcutaneous injection of ciVAX E. coli $(n=5)$, ciVAX E. cloacae $(n=10)$ or ciVAX sham $(n=5)$, followed by challenge with $5 \times 10^{6}$ c.f.u. E. coli RS218 35 days after the vaccination.

captured PAMPs was required for protection-the survival rate was only $17 \%$ in the sham mice, which received the same biomaterial scaffold containing GM-CSF and CpG adjuvant but without FcMBL bead-captured E. coli PAMP antigens (Fig. 3a). Post-mortem quantification of bacteria within isolated organs revealed that the unvaccinated and sham-treated mice had a mean of $>1 \times 10^{8}$ colony-forming units (c.f.u.) per gram in the lung, liver, kidney and spleen, whereas vaccination reduced these numbers by $10^{5}-10^{7}$ (Fig. 3b) and there was a significant increase in specific anti-RS218 antibodies in the vaccinated animals (Fig. 3c). Moreover, the ciVAX generated a durable immune response, as a single injection protected $>90 \%$ of mice from a lethal RS218 challenge delivered 90 days after the vaccination (Fig. 3d) with significant decreases in the bacterial counts in organs on post-mortem analysis (Fig. 3e) and a durable increase in the levels of antibody to RS218 (Fig. 3f). Importantly, the infection vaccine did not substantially change the gut microbiome of rabbits over a period of 46 days after vaccination with E. coli or MRSA ciVAX (Supplementary Fig. 4a-c). We next tested a homologous vaccine in a pig model of Gram-negative septic shock involving intravenous injection of a human clinical isolate of E. coli 41949 (OM:H26), which leads to rapid renal failure and death ${ }^{23}$. The animals were vaccinated with $16 \times$ the mouse dose (112 ng), boosted with $4 \times$ the mouse dose ( $28 \mathrm{ng}$ ) on day 28 with 41949 ciVAX and then challenged with E. coli 41949 on day 42. The four untreated animals developed neutropaenia, thrombocytopaenia, bacteraemia, elevated lactate levels, acute kidney injury with extremely high creatinine levels and swine-specific Sequential Organ Failure Assessment (ssSOFA) ${ }^{23}$, indicating fulminant sepsis within $12 \mathrm{~h}$, which required that they be euthanized (Fig. $3 \mathrm{~g}$ ). In contrast, the four vaccinated pigs had increased neutrophil counts, low lactate levels, normal SOFA scores and creatinine levels below the euthanasia threshold (Fig. 3h,i and Supplementary Fig. 5); and they all survived to the end of the experiments at $28(n=2)$ and $72 \mathrm{~h}$ $(n=2)$, respectively (Fig. $3 \mathrm{~g}$ ). Importantly, the vaccinated pigs were clinically healthy, despite live pathogens and dead pathogen-derived PAMPs remaining in their bloodstream (Supplementary Fig. 5), suggesting that the ciVAX vaccine protected the pigs from endotoxic shock through a strengthened humoral and cellular immune response rather than a bactericidal mechanism. There was a transient increase in serum interleukin-6 levels during the E. coli challenge infusion (Supplementary Fig. 5) but otherwise there were no notable changes in the animals.

ciVAX can be based on other biomaterial scaffolds and requires all agents. Although the studies described up to this point were performed with one biomaterial vaccine system (MPS rods), this general approach is likely to be useful with a variety of material-based vaccine strategies. To test this possibility, PAMPs bound to FcMBL beads were combined with another biomaterial-based vaccine that is currently in a clinical trial for the treatment of Stage IV melanoma (WDVAX; NCT01753089). Vaccination with PLG ciVAX containing cefepime-killed RS218 captured on FcMBL beads promoted 


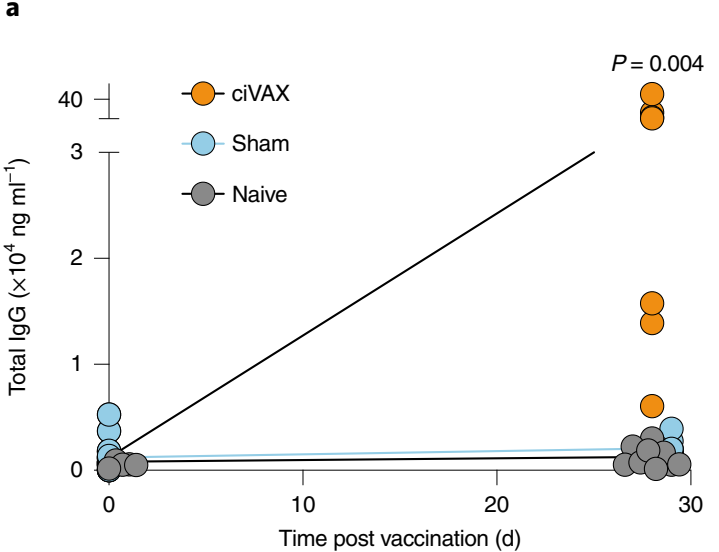

b

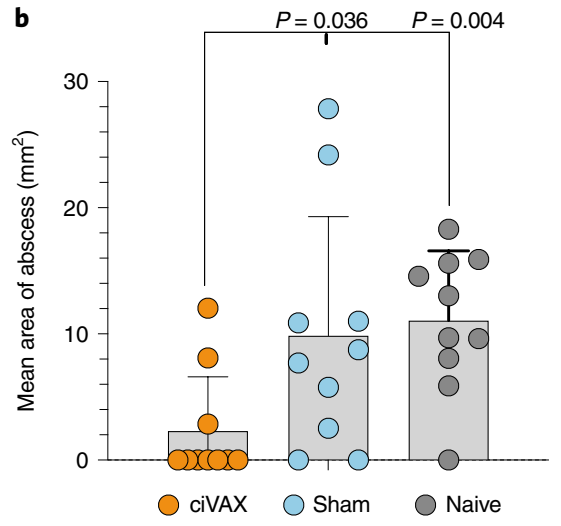

Fig. 5 | ciVAX provides functional immune protection against Gram-positive S. aureus. The ciVAX vaccine was produced by mixing lyophilized MPS biomaterial scaffold containing murine GM-CSF and CpG with vancomycin- and daptomycin-killed MRSA (ATCC 12598) captured on FcMBL paramagnetic beads. a, Female BALB/c mice (8-10 weeks old) were vaccinated and boosted (day 14) by subcutaneous injection with ciVAX, sham scaffold without FcMBL-RS218, or unvaccinated control (naive; $n=10$ per group). MRSA-specific antibodies in the mouse serum were measured using vancomycinand daptomycin-killed MRSA adsorbed directly on the well plates, blocked to reduce interaction between staphylococcal protein A and IgG. $\mathbf{b}$, The mice were challenged with $5 \times 10^{7}$ c.f.u. MRSA on the left flank 35 days after vaccination and monitored for abscess formation for a further $72 \mathrm{~h}$. Data are the mean \pm s.d. Statistically significant differences between the independent groups were identified using a non-parametric Mann-Whitney $U$ test.

survival in a high fraction of mice that were subsequently challenged 35 days post vaccination (Fig. $4 \mathrm{a}$ ) and dramatically reduced the bacterial titres in all of the analysed organs (Fig. 4b). The vaccines that were fabricated without GM-CSF and CpG or PAMP and FcMBL, or that were missing all four active ingredients provided no survival benefit and led to high bacterial loads in the organs of the challenged mice (Fig. 4a,b and Supplementary Fig. 7). These findings indicate that both the dendritic-cell accumulation/activation function of the scaffold component of ciVAX and the antigen capture via FcMBL is required for the efficacy of these vaccines. The mice that were challenged 90 days post vaccination with the PLG ciVAX also demonstrated high survival rates (Fig. 4c), which provides support for the durability of the induced immune responses. We next examined the ability of PAMPs captured from a single pathogen (Enterobacter cloacae) to confer cross protection from a challenge from another Enterobacteriaceae (E. coli). PAMPs collected from $E$. cloacae were able to protect $77 \%$ of the mice that were challenged with a lethal dose of E. coli (Fig. 4d), indicating the PAMPs captured include conserved epitopes that provide a broad spectrum of protection. As the PLG-based ciVAX, unlike the MPS rods, requires surgical implantation, all subsequent studies were performed with the MPS-based ciVAX.

S. aureus ciVAX prophylaxis reduces skin abscesses in mice. $S$. aureus is the most common Gram-positive organism causing sepsis and many $S$. aureus strains are antibiotic resistant, yet there is no approved vaccine for this pathogen ${ }^{24}$. Mice immunized with ciVAX containing PAMPs isolated from MRSA mounted potent, durable and specific IgG responses that were multiple-fold higher than the sham group (Fig. 5a). Vaccinated mice that were subsequently challenged with MRSA infection demonstrated significantly fewer skin abscesses compared with the controls (Fig. 5b).

Potential for ciVAX use in pandemic applications. The broad-spectrum binding capabilities of FcMBL could enable the development of vaccines from PAMPs extracted from the blood or tissues of infected individuals. In veterinary medicine these could qualify as Autogenous Biologics for approval by USDA-APHIS (9 CFR 113.113). In one-world health and pandemic applications, the ciVAX technology could be used in ring vaccination to protect others in the community from infection by the original pathogen or even by closely related species and variants, potentially providing benefit even when there is insufficient time for full identification of the organism. As it was impractical to prepare ciVAX from mice due to their limited blood volumes, to model ring vaccination, PAMPs were magnetically captured from the blood of a pig previously infected with E. coli 41949 (OM:H26) in less than 1 h using FcMBL beads, which were then combined with the lyophilized biomaterial components. After this single heterologous vaccination with captured E. coli OM:H26 PAMPs, we challenged the mice with a lethal dose of a different $E$. coli serotype (O18:H7). All of the unvaccinated mice died following challenge with $E$. coli RS218 (O18:H7) and their organ bacterial counts were $>1 \times 10^{8}$ c.f.u. $\mathrm{g}^{-1}$ (Fig. 6a,b). In contrast, $80 \%$ of the vaccinated mice survived the lethal E. coli RS218 challenge and there was a significant decrease in pathogen load in the lungs and kidneys at the end of the 28-day study as well as a trend towards decreased levels of pathogen in the spleen and lungs (Fig. 6a,b).

\section{Discussion}

There are no approved vaccines for the most common pathogens causing sepsis. A four-valent vaccine comprising the E. coli $\mathrm{O} 1 \mathrm{~A}$, $\mathrm{O} 2$, $\mathrm{O} 6$ and $\mathrm{O} 25 \mathrm{~B}$ antigens conjugated to Pseudomonas aeruginosa exoprotein $\mathrm{A}$ is in Phase 2 trials for the prevention of extra-intestinal E. coli infections ${ }^{25}$. Although this vaccine led to an increase in functional antibodies to at least two of the four antigens, it is estimated that more than ten different antigens will be needed to cover more than $60 \%$ of the extra-intestinal E. coli and $90 \%$ of meningitis isolates. There are no approved prophylactic or therapeutic vaccines for $S$. aureus or MRSA infection. There have been attempts to develop vaccines to $S$. aureus polysaccharide type 5 or 8 conjugates but these have not been successful in clinical trials and the more recent trials have again focused on increasing the number of antigens ${ }^{24}$. We anticipate that ciVAX could be more effective than these approaches because multiple antigens (including glycoproteins and glycolipids) are included in their native forms, antigens are presented on particulate substrates and are slowly released from the biomaterial depots-thereby yielding strong immunogenicity with little toxicity and adverse events-and ciVAX includes bioactive molecules that recruit large numbers of dendritic cells for in vivo priming and maturation, which promote the development of potent affinity-matured immune responses. 

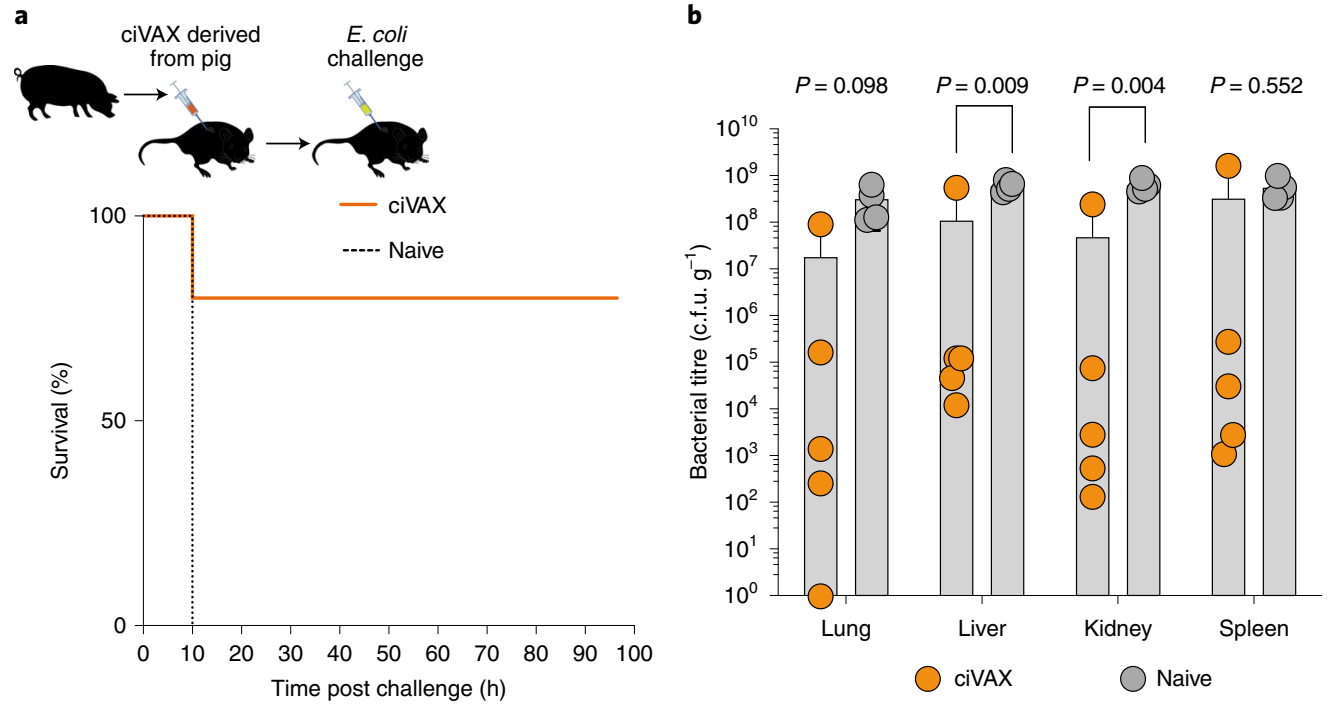

Fig. 6 | ciVAX produced from an E. coli-infected animal could be used to protect against another $E$. coli serotype. The ciVAX vaccine was produced by capturing E. coli 41949 (OM:H26) PAMPs directly from the blood of an infected pig treated with cefepime, using FcMBL-coated superparamagnetic beads, and then mixed with lyophilized MPS biomaterial scaffold containing murine GM-CSF and CpG. $\mathbf{a}$, Mice were unvaccinated (naive) or vaccinated ( $n=5$ mice per group), challenged with lethal doses of E. coli RS218 (O18:H7) on day 35 and followed for $96 \mathrm{~h}$. $\mathbf{b}$, The mice were killed at humane endpoints or the end of the experiment and the pathogens in lung, liver, spleen and kidney were measured by quantitative culture on sheep blood agar. Data are the mean \pm s.d. Significant differences were identified using an unpaired Student's $t$-test.

We used two biomaterial systems and although they both provide sustained presentation of GM-CSF, CpG and antigens, there are some differences between the two. GM-CSF release from both systems follows similar kinetics, with sustained release over approximately $7-10$ days in vitro ${ }^{16,17}$. In vitro, the release of $\mathrm{CpG}$ and antigen is slower from PLG than from MPS. This probably relates to the PLG fabrication process, which results in the encapsulation of these agents, with their release at least partially dependent on the slow degradation of the PLG. In contrast, these agents are surface-absorbed to the MPS, which may lead to more rapid release owing to both dissolution and to the more rapid degradation of the MPS (weeks versus months for MPS versus PLG). Applications in which release over months (rather than weeks) is desired may benefit from the PLG system. It may also be possible to use ciVAX without pre-loading PAMPs to the FcMBL in slow-progressing infections and instead allow PAMPs present in the infected animal to bind to the FcMBL in situ. This concept has some overlap with recent work in the cancer field demonstrating that a biomaterial-based therapeutic vaccine without antigen can be combined with chemotherapy, which leads to immunogenic cell death and antigen generation in situ, yielding a potent immune response ${ }^{26}$.

The benefit of ciVAX containing multiple (polyclonal) antigens (rather than a subset of immunodominant antigens expressed using recombinant DNA technology) may be a limitation, as the PAMP material is not defined and the antigens have not all been identified. It is possible that ciVAX could contain antigens causing immunosuppressive reactions rather than protective immune responses. If isolating PAMPs from an infected individual for ring immunization, although the majority of captured PAMPs are expected to be associated with the target pathogen, it is possible that PAMPs present from earlier infections could be captured and presented in the ciVAX. Furthermore, the impact of cellular versus humoral immunity needs to be determined and further studies are needed to determine how ciVAX will work in those with immature or weak immune systems. The large-animal sepsis experiment needs additional immunoprofiling and the measurement of additional cytokines, which will be addressed in future studies. It will also be important to explore therapeutic vaccinations of this technology to assess its potential clinical utility.

The inclusion of GM-CSF and CpG enhances the accumulation and activation of dendritic cells at the injection site as well as the number of dendritic, $\mathrm{T}$ and $\mathrm{B}$ cells in the draining lymph node. These findings are consistent with our previous work showing that the release of GM-CSF and CpG leads to a significant increase in activated dendritic cells at the injection site ${ }^{16,17,27}$ and also enhanced antigen presentation ${ }^{28,29}$. A notable observation from this work was the rapidity of the increase in the number of $\mathrm{CD} 4^{+}$and $\mathrm{CD} 8^{+}$ $\mathrm{T}$ cells in the vaccine-draining lymph nodes following vaccination with ciVAX. We examined host responses to ciVAX and the kinetics of immune cell homing, retention and interactions in the lymph nodes, and found that several factors could contribute to this outcome. Due to the ubiquitous nature of E. coli antigen(s), a pool of pre-existing memory $\mathrm{T}$ cells to antigens in ciVAX may exist in the animals, leading to a rapid expansion of pre-existing T-cell populations following vaccination. The combination of biomaterial (MPS), a mixture of FcMBL-captured antigens (including lipopolysaccharide), a danger signal ( $\mathrm{CpG}$ ) and an APC factor (GM-CSF) may also lead to immune responses that are faster than previously observed. Naive $\mathrm{CD}^{+} \mathrm{T}$ cells are rich in CCR7 and it has been recognized that this provides homing cues for T-cell recruitment and retention in the lymph nodes ${ }^{30}$ in response to the production of CCL 21 by stromal cells of the lymph node following inflammatory signals. Under normal healthy conditions, the T-cell entry and exit rates are coordinated to maintain lymphocyte homeostasis in blood and in the secondary lymphoid organs, although the mechanistic basis for this coordination is still being investigated. However, the normal rate of approximately 5,000 $\mathrm{T}_{\text {cells }} \mathrm{h}^{-1}$ moving through a lymph node ( ref. $^{31}$ ) may possibly increase to a much higher magnitude in an inflamed lymph node following ciVAX administration. Furthermore, inflammatory cues within a lymph node may quickly upregulate CD69 expression on $\mathrm{CD}^{+} \mathrm{T}$ cells that can bind to S1PR1 at the cell surface and enhance the T-cell dwell time within such lymph nodes. It is also possible that some of the carbohydrate-containing antigens in ciVAX, such as post-translationally modified glycoproteins, 
behave as super-antigen-like moieties that can activate as much as $20 \%$ of the T cells without strict T cell-receptor specificities (unlike $<0.0001 \%$ in a $\mathrm{T}$ cell receptor-specific manner $)^{32}$.

In summary, the ciVAX PAMP vaccination technology can be used to mount strong immune responses to both Gram-negative and Gram-positive bacteria following subcutaneous administration. The system produces life-saving effects in small and large-animal models of sepsis caused by infection with $E$. coli from different serotypes. The broad-spectrum efficacy against MRSA and multidrug-resistant bacteria suggest that this technology could represent an alternative to antibiotics. Importantly, given the broad-spectrum PAMP-binding capabilities of $\mathrm{FcMBL}^{14}$, this vaccine approach should also be effective against a wide range of glycosylated fungal, parasitic and viral targets, including the severe acute respiratory syndrome coronavirus 2 spike protein. Moreover, the modular nature of the technology and the use of lyophilized components should make this vaccination particularly useful for global-health applications. The ability to target PAMPs from a broad spectrum of pathogens without time-consuming culturing steps also raises the possibility that it could provide a clinical countermeasure to quickly control the spread of infection in pandemics and biothreat scenarios. The ciVAX technology could be manufactured at a large scale as both of the compounds (MPS and GM-CSF) are currently produced at a relatively large scale, as are many adjuvants that could be used in vaccine manufacturing. The manufacture of the bacterial lysate could be scaled using existing microbial fermentation technologies for providing antigens. The actual process used to fabricate the vaccines is an aqueous-based admix procedure that could be scaled to support a response to a pandemic. The final vaccine could be packaged using existing fill-and-finish technology. The entire vaccine could be fabricated and stored, or the ciVAX system without an antigen might be stable enough to be prefabricated from GMP-sourced components and stored in lyophilized form at strategically located depots. The antigen source, once identified and manufactured, could be added to the stored antigen-free vaccine before delivery or even at the bedside. PAMPs collected from a previously infected individual could potentially be used in this scenario, as long as the collected material was stored appropriately to avoid degradation.

\section{Methods}

Preparation of bacterial antigens. S. aureus 12598 (ATCC 12598), E. coli RS218 (provided by J. R. Johnson from the University of Minnesota) and E. coli 41949 (clinical isolate 41949 obtained from the Brigham and Women's Hospital, Crimson Biorepository) were cultured individually in RPMI medium containing $10 \mathrm{mM}$ glucose at $37^{\circ} \mathrm{C} 225$ r.p.m. to a McFarland of 1.0 (approximately $2 \times 10^{8}$ c.f.u. $\mathrm{ml}^{-1}$ ). The cultures were placed on ice for $10 \mathrm{~min}$ to slow growth and then centrifuged at $3,000 \mathrm{~g}$ for $10 \mathrm{~min}$ at $4^{\circ} \mathrm{C}$ (Eppendorf 5810R). The supernatants were discarded and the pellets were resuspended in fresh RPMI-10 mM glucose until a McFarland of 5.0 (approximately $1 \times 10^{9}$ c.f.u. $\mathrm{ml}^{-1}$ ) was achieved. Next, $1 \mathrm{mg} \mathrm{ml}^{-1}$ cefepime (NDC 25021-121-20) was added to the E. coli, and $1 \mathrm{mg} \mathrm{ml}^{-1}$ vancomycin (NDC 0409-4332-49) and $0.5 \mathrm{mg} \mathrm{ml}^{-1}$ daptomycin (Toronto Research Chemicals) were added to $S$. aureus. All cultures were then placed back in the incubator overnight (approximately $17 \mathrm{~h}$ ) at $37^{\circ} \mathrm{C}$ and 225 r.p.m. The following morning, each culture was passed through a $70-\mu \mathrm{m}$ cell strainer (Sigma-Aldrich) to remove the large cellular debris. A second dose of the respective antibiotic was added to each culture and incubated overnight (approximately $17 \mathrm{~h}$ ) at $37^{\circ} \mathrm{C}$ and 225 r.p.m. Each culture was then plated in triplicate on trypticase soy agar II with $5 \%$ sheep blood (Becton Dickinson) and placed at $37^{\circ} \mathrm{C}$ in a $5 \% \mathrm{CO}_{2}$ incubator for $24 \mathrm{~h}$ to confirm complete bacterial death by the absence of c.f.u. The cultures were then stored a $-80^{\circ} \mathrm{C}$. When required, the cultures were thawed and diluted 1:2 in $50 \mathrm{mM}$ Tris- $\mathrm{HCl}$, $150 \mathrm{mM} \mathrm{NaCl}, 0.05 \%$ Tween-20 and $5 \mathrm{mM} \mathrm{CaCl}_{2}, \mathrm{pH} 7.4$ (TBST-5 $\mathrm{mM} \mathrm{CaCl}_{2}$; Boston BioProducts). FcMBL beads ${ }^{13}\left(5 \mathrm{mg} \mathrm{ml}^{-1}\right.$ stock; biotinylated FcMBL on $1 \mu \mathrm{M}$ Dyna MyOne Dynabeads (Thermo Fisher Scientific)) were added at $25 \mu \mathrm{ml}^{-1}$ and the solution was mixed at room temperature for 30 min using a HulaMixer (Thermo Fisher Scientific) set to 20 r.p.m. After FcMBL capture/enrichment of bacterial PAMPs, the solution was magnetized (Thermo Fisher Scientific) for $10 \mathrm{~min}$ to allow the removal of supernatant without bead disruption. The tubes were removed from the magnet and an equal volume of fresh TBST-5 $\mathrm{mM} \mathrm{CaCl}_{2}$ was added to wash the beads. Finally, the tubes were inverted until the beads were completely homogenized. This solution was stored at $-80^{\circ} \mathrm{C}$ until needed.
Quantification of bacterial antigens captured on FcMBL beads. The captured PAMP bacterial antigens captured on FcMBL beads were quantified using an enzyme-linked lectin-sorbent assay as previously published ${ }^{13}$. Briefly, FcMBL was biotinylated at the amino terminus of the Fc protein using an N-terminal amino-oxy reaction and then coupled to streptavidin superparamagnetic beads ( $1 \mu \mathrm{M}$ MyOne Dynabeads; Thermo Fisher Scientific). The samples were screened using $5 \mu \mathrm{g}$ of the FcMBL beads, $200 \mu \mathrm{l}$ test sample and $800 \mu \mathrm{l}$ TBST- $5 \mathrm{mM} \mathrm{CaCl}_{2}$ supplemented with $10 \mathrm{mM}$ glucose, incubated for $20 \mathrm{~min}$ at $22^{\circ} \mathrm{C}$ and 950 r.p.m. in a plate shaker (Eppendorf). The automated magnetic-handling system (KingFisher Flex; Thermo Fisher Scientific) was used for washing steps, followed by colorimetric development with horseradish peroxidase-labelled MBL. The enzyme-linked lectin-sorbent assay was quenched using sulfuric acid, and the optical density at $450 \mathrm{~nm}$ was read and compared with a standard curve of mannan, a well-known yeast PAMP.

Preparation of vaccine scaffolds. MPS rods. To synthesise high-aspect-ratio MPS $(88 \mu \mathrm{m} \times 4.5 \mu \mathrm{m}), 4 \mathrm{~g}$ P123 (Sigma-Aldrich) surfactant (average $M_{\mathrm{n}}$ of approximately 5,800 ) was dissolved in $150 \mathrm{ml}$ of $1.6 \mathrm{M} \mathrm{HCl}$ solution, stirred with 8.6 g tetraethyl orthosilicate $\left(98 \%\right.$; Sigma-Aldrich) at $40^{\circ} \mathrm{C}$ for $20 \mathrm{~h}$, followed by incubation at $100^{\circ} \mathrm{C}$ for $24 \mathrm{~h}$. To extract the surfactant, the as-synthesised particles were refluxed for $10 \mathrm{~h}$ in $1 \% \mathrm{HCl}$ in ethanol. The resulting MPS particles were then filtered, washed with ethanol and dried. The MPS morphology was measured using optical microscopy. To make the completed MPS vaccine, $3 \mu \mathrm{l}$ GM-CSF (murine GM-CSF, Peprotech; human-leukine for pig and rabbit, Sanofi) and $100 \mu \mathrm{l} \mathrm{CpG}$ (murine CpG, ODN 1826; human CpG ODN 2006 for pig and rabbit, InvivoGen) were both added at $1 \mathrm{mg} \mathrm{ml}^{-1}$ in water for injection. Captured antigen, defined antigen or lysate (for example, $25 \mu \mathrm{FcMBL}$ beads with captured 15 PAMP units of enriched E. coli lysate) were then added to the $10 \mathrm{mg}$ MPS solution. The resulting slurry was sonicated for $5-10 \mathrm{~s}$ in a water bath and mixed overnight at room temperature using a HulaMixer (Thermo Fischer Scientific) at 45 r.p.m. The mixture was then frozen at $-80^{\circ} \mathrm{C}$ for a minimum of $12 \mathrm{~h}$ before lyophilization. Lyophilization was performed for a minimum of $48 \mathrm{~h}$. The material was reconstituted in water for injection before injection.

Production of ciVAX directly from the blood of infected animals. A pig was infused with E. coli as described in the 'Animal models' section as per Institutional Animal Care and Use Committee protocol no. 17-03-3400. Briefly, a bolus of $1.2-1.5 \times 10^{5}$ c.f.u. $\mathrm{kg}^{-1}$ E. coli 41949 was administered to an anaesthetized animal via the femoral vein at a rate of $1 \mathrm{ml} \mathrm{min}^{-1}$ over $10 \mathrm{~min}$ using a syringe pump. Following the bolus, the femoral line was pulled, the animals were recovered from anaesthesia and returned to housing. Once recovered, the animals received a second infusion of $3.51 \times 10^{7}-1.36 \times 10^{8}$ c.f.u. $\mathrm{kg}^{-1}$ E. coli 41949 via the right jugular vein over $4 \mathrm{~h}$ using a syringe pump. A single intravenous injection of $20 \mathrm{mg} \mathrm{kg}^{-1}$ cefazolin was administered $2 \mathrm{~h}$ after the start of the infusion. At the end of the infusion, $2 \mathrm{ml}$ heparinized pig blood was mixed for 30 min with $250 \mu \mathrm{FcMBL}$ beads in Tris-buffered saline with Tween-20 and $5 \mathrm{mM}$ calcium. The beads and captured PAMPs were collected on a magnet, washed with Tris-buffered saline with Tween-20 and $5 \mathrm{mM}$ calcium, and mixed with MPS as described earlier.

PLG acid. PLG acid microspheres (18 mg; Phosphorex) containing $9 \mu \mathrm{g}$ encapsulated GM-CSF (murine GM-CSF, Sanofi; human-leukine for pig and rabbit, Peprotech) were combined with a 1-ml solution of captured antigen, defined antigen or lysate (for example, $25 \mu \mathrm{FcMBL}$ beads with captured 15 PAMP units of enriched $E$. coli lysate) vortexed for $5 \mathrm{~min}$ before being flash frozen with liquid $\mathrm{N}_{2}$ and lyophilized for $72-96 \mathrm{~h}$. The lyophilized powder was combined with $300 \mu \mathrm{g}$ CpG (murine CpG, ODN 1826; porcine, rabbit, human CpG, ODN 2006 for pig and rabbit, InvivoGen), which had been condensed with polyethyleneimine and $130 \mathrm{mg}$ sieved sucrose $(250-450 \mu \mathrm{m})$, and then manually mixed until homogenous. This homogenous powder was poured into a die set and formed using a Carver press under $1,500 \mathrm{psi}$ of pressure for $45 \mathrm{~s}$, resulting in a solid disc that was approximately $2 \mathrm{~mm}$ high and $9 \mathrm{~mm}$ in diameter. The disc was placed in a pressure chamber (Parr) overnight and exposed to 800 psi $\mathrm{CO}_{2}$ (approximately $18 \mathrm{~h}$ ). Before implantation, the disk was placed in $10 \mathrm{ml}$ of water for injection (Thermo Fisher Scientific), which dissolves the sieved sucrose, creating the final porous structure for implantation. This step reduces the vaccine dose by approximately $50-60 \%$.

Quantitation of GM-CSF, CpG and endotoxin release. CpG was measured by HPLC SEC using an Xterra C18 column in TEAA/acetonitrile buffers on an Agilent 1260 II system. The endotoxin samples were run on an Endosafemulti-cartridge system (Charles River Laboratories) using limulus lysate (LAL) test cartridges (Charles River Laboratories, PTS55F). The GM-CSF samples were quantified using an R\&D DuoSet mouse GM-CSF kit (DY415) according to the manufacturer's instructions.

SDS-PAGE. Samples were run on a Novex 4-20\% Tris-glycine mini-protein gels (Invitrogen, cat. no. EC60252) using Tris-glycine SDS running buffer (Invitrogen, cat. no. LC2675-4), stained with Coomassie Fluor orange protein gel stain (Thermo Fisher Scientific, C-33250) and silver stain (Pierce, cat. no. 24612) according to the manufacturer's instructions. Molecular weights were estimated based on a BioRad ladder (cat. no. 131-0363). 
Mass spectrometry. Sample preparation procedure. All samples were in PBS buffer. After liquid removal, the samples were dissolved in $50 \mu \mathrm{l}$ of $50 \mathrm{mM}$ triethylammonium bicarbonate buffer, followed by trypsin (Promega) digestion for $3 \mathrm{~h}$ at $38^{\circ} \mathrm{C}$. The digested samples were dried to $20 \mu \mathrm{l}$ and half $(10 \mu \mathrm{l})$ was submitted for mass spectrometry analysis.

Mass spectrometry analysis. The LC-MS/MS experiment was performed on a Lumos Tribrid Orbitrap mass spectrometer (Thermo Fischer) equipped with an Ultimate 3000 (Thermo Fisher Scientific) nano-HPLC. Peptides were separated onto a $150-\mu \mathrm{m}$-inner-diameter microcapillary trapping column packed first with approximately $2 \mathrm{~cm}$ of C18 Reprosil resin $(5 \mu \mathrm{m}, 100 \AA$; Dr. Maisch $\mathrm{GmbH})$, followed by a PharmaFluidics $50-\mathrm{cm}$ analytical column. Separation was achieved by applying a gradient of $5-27 \%$ acetonitrile in $0.1 \%$ formic acid over $90 \mathrm{~min}$ at $200 \mathrm{nl} \mathrm{min}^{-1}$

Electrospray ionization was enabled through the application of a voltage of $2 \mathrm{kV}$ using a home-made electrode junction at the end of the microcapillary column and sprayed from metal tips (PepSep). The mass spectrometry survey scan was performed in the Orbitrap in the range of 400 to $1,800 \mathrm{~m} / z$ at a resolution of $6 \times 10^{4}$, followed by the selection of the twenty most-intense ions (TOP20) for collision-induced dissociation (CID) tandem mass spectrometry $\left(\mathrm{MS}^{2}\right)$ fragmentation in the ion trap using a precursor isolation width window of $2 \mathrm{~m} / z$, automatic gain control (AGC) setting of 10,000 and a maximum ion accumulation of $100 \mathrm{~ms}$. Singly charged ion species were not subjected to collision-induced-dissociation fragmentation. The normalized collision energy was set to $35 \mathrm{~V}$ and an activation time of $10 \mathrm{~ms}$. Ions in a $10 \mathrm{ppm} \mathrm{m} / z$ window around ions selected for LC-MS/MS were excluded from further selection for fragmentation for $60 \mathrm{~s}$

Data analysis. Raw data were submitted for analysis in the Proteome Discoverer 2.4 (Thermo Fisher Scientific) software. Assignment of the LC-MS/MS spectra was performed using the Sequest HT algorithm by searching the data against a protein sequence database including all of the entries from our Uniprot_EColi and Uniprot_SAureus databases as well as other known contaminants such as human keratins and common laboratory contaminants. Quantitative analyses between samples were performed by label-free quantitation between different sets of samples. Sequest HT searches were performed using a 10-ppm precursor ion tolerance and requiring the amino/carboxy termini of each peptide to specifically adhere to trypsin protease, while allowing up to two missed cleavages. Methionine oxidation $(+15.99492 \mathrm{Da})$ was set as variable modification. An $\mathrm{MS}^{2}$ spectra-assignment false-discovery rate of $1 \%$ at both the protein and peptide level was achieved by applying the target-decoy database search using Percolator.

Construction of bound cellular location treemaps. For the construction of the Bound Cellular Location Treemaps, the R package Treemap was utilized ${ }^{33}$. The treemap visualizes the hierarchical number of proteins that were found in a given subcellular location as a set of nested rectangles. The labelled rectangles represent the subcellular location and the area represents the relative number of proteins found in each location. Determination of location was by the presence or absence of proteins found in the proteomics data comparing the lysate and beads label-free-quantitation proteomics. Subcellular location was determined using the Uniprot database with respect to species.

Construction of bound cellular location word clouds. For the construction of the bound cellular location word clouds, the $\mathrm{R}$ package wordcloud 2 was applied ${ }^{34}$. The word clouds represent the location of the proteins that were bound to the beads using a visual representation of the location labels. The size of the text in the word clouds are representative of the relative number of proteins found in a given subcellular location. Determination of location was by the presence or absence of proteins found in the proteomics data comparing the lysate and beads label-free-quantitation proteomics. Subcellular location was determined using the Uniprot database with respect to species.

Animal models. Mice. All studies were performed in accordance with institutional guidelines and approved by the Institutional Animal Care and Use Committee of Harvard Medical School. Male and female BALB/c mice (8-10 weeks old, weighing 18-25 g; Charles River Laboratories) were used in the experiments and housed in a biosafety level 2 room under standard conditions. The animals were provided autoclaved bedding and enviro-dri nesting material, had free access to food and water, and were kept on a $12 \mathrm{~h}$ dark-12 h light cycle.

Vaccination. The mice were briefly anaesthetized, their fur was clipped and $250 \mu \mathrm{l}$ vaccine (vortexed before injection) was subcutaneously injected into the dorsum or flank. The vaccine sites were monitored over time for irregularities. Serum was collected from the mice at 14-day intervals. The mice that were vaccinated with ciVAX MRSA were given a booster of ciVAX MRSA on day 14.

RS218 and MRSA challenges. Male and female BALB/c mice were intraperitoneally challenged with $5 \times 10^{6}$ c.f.u. E. coli (RS218) in $100 \mu$. The animals were monitored several times per day for mortality and humane criteria. Animals presenting as moribund were euthanized and considered deceased. Female BALB/c mice were challenged with $5 \times 10^{7}$ c.f.u. MRSA on the left flank on day 35 . The animals were briefly anaesthetized, their fur was clipped, depilatory cream was applied and the pathogen (in $50 \mu \mathrm{l}$ ) was injected subcutaneously. The animals were monitored daily for lesion size and progression. Sustained release buprenorphine and fluids were provided for all challenge models. At termination, organs were extracted for immunological and c.f.u. quantification.

Rabbits. Approval for the study was obtained from the Institutional Animal Care and Use Committee (PARF). E. coli RS218 was cultured in RPMI-10 mM glucose medium at $37^{\circ} \mathrm{C}$ and 220 r.p.m. until a McFarland of $0.5\left(1 \times 10^{8}\right.$ c.f.u. $\left.\mathrm{ml}^{-1}\right)$ was achieved. The culture was then centrifuged at $3,000 \mathrm{~g}$ for $15 \mathrm{~min}$ at $4^{\circ} \mathrm{C}$ to pellet the bacteria. The pellet was resuspended in RPMI-10 mM glucose until a McFarland of $5.0\left(1 \times 10^{9}\right.$ c.f.u. $\left.\mathrm{ml}^{-1}\right)$ was achieved. The antibiotic, ceftriaxone (NDC 60505$6104-4)$, was added at $1 \mathrm{mg} \mathrm{ml}^{-1}$ and the culture was incubated overnight at $37^{\circ} \mathrm{C}$ and 220 r.p.m. The cellular debris was removed by filtering through a $70-\mu \mathrm{m}$ membrane the following day and the culture was treated again with $1 \mathrm{mg} \mathrm{ml}^{-1}$ ceftriaxone overnight. The culture was then plated in duplicate on trypticase soy agar II with $5 \%$ sheep blood to ensure complete bacterial death, which was confirmed by the absence of c.f.u. after an overnight incubation at $37^{\circ} \mathrm{C}$. To make the $50 \%$ lysate solution, the culture was diluted $1: 2$ in TBST-5 $\mathrm{mM} \mathrm{CaCl}_{2}$. To make the $1: 2$ bead-captured solution, the culture was diluted $1: 2$ in TBST- $5 \mathrm{mM} \mathrm{CaCl}_{2}$ and $1 \mu \mathrm{m}$ FcMBL beads were added at a concentration of $25 \mu \mathrm{ml}^{-1}$. This solution was then incubated at room temperature for $30 \mathrm{~min}$ using end-over-end mixing. Beads with captured PAMPs were magnetized for $5 \mathrm{~min}$ and the supernatant was removed. An equal volume of TBST $-5 \mathrm{mM} \mathrm{CaCl}_{2}$ was added back and the beads were inverted to homogenize in solution. Three rabbits were injected by subcutaneous route using either the $50 \%$ whole-cell lysate or the 1:2 bead-captured solution containing equivalent PAMP levels $\left(22.9 \pm 1.9\right.$ and $20.5 \pm 4.2$ PAMPs ml $^{-1}$, respectively). Rabbits were boosted at day 28 with the same preparations used for priming. At the conclusion of the study at day 42 , the vaccination sites were compared with regards to gross pathology and histology.

Pigs. Approval for the study was obtained from the Institutional Animal Care and Use Committee (protocol no. 17-03-3400). The work was conducted at an AAALAC-accredited and USDA-registered facility and in accordance with NIH guidelines. Outbred juvenile Yorkshire swine $(40-50 \mathrm{~kg})$ were vaccinated against E. coli 41949 via two subcutaneous injections of $4 \mathrm{ml}$ in the left or right flank. The initial vaccination occurred 43 days before the challenge and was $16 \times$ the mouse dose. The animals received a booster vaccine on day 27/28. The booster was given as a single subcutaneous injection of $4 \mathrm{ml}$ on the left flank and was $4 \times$ the mouse dose.

On day 43 , the animals were anaesthetized with intramuscular injections of atropine $\left(0.04 \mathrm{mg} \mathrm{kg}^{-1}\right)$, telazol $\left(4.4 \mathrm{mg} \mathrm{kg}^{-1}\right)$ and xyalzine $\left(2.2 \mathrm{mg} \mathrm{kg}^{-1}\right)$, and maintained on isoflurane $(1.5-2.0 \%)$ and oxygen $\left(0.5-2.01 \mathrm{~min}^{-1}\right)$ delivered through an $8.5-9-\mathrm{mm}$ endotracheal tube using a positive-pressure ventilator. The animals were placed in the supine position and a 20 -g intravenous cannula was placed in the left or right marginal ear vein for the administration of drugs and fluids, and sterile saline $(0.9 \% \mathrm{NaCl})$ was administered continuously at a rate of $200-300 \mathrm{mlh}^{-1}$. A 10-12-Fr Foley catheter (SurgiVet, Smiths Medical) was placed for urinary drainage and a 5-6-Fr percutaneous sheath catheter (Arrow, Teleflex) was placed in the left or right femoral vein for obtaining a blood sample before surgery to serve as a baseline and for pathogen administration after surgery. The ventilator settings were determined on an individual basis depending on the size of the pig.

Bilateral neck cutdowns were performed to expose and isolate the right carotid artery and the left and right jugular veins. The right carotid and right jugular were cannulated with an 8-Fr sheath catheter (Arrow). In two animals, the left jugular was cannulated with a 7-Fr triple lumen catheter (Arrow) to allow for interventions, if needed. After the cannulations were complete, the incisions were closed. The animals remained under anaesthesia and a bolus of $1.2-1.5 \times 10^{5}$ c.f.u. $\mathrm{kg}^{-1}$ E. coli 41949 was administered via the femoral vein at a rate of $1 \mathrm{ml} \mathrm{min}-1$ over $10 \mathrm{~min}$ using a syringe pump. Following the bolus, the femoral line was pulled, the animals were recovered from anaesthesia and returned to housing. Once recovered, the animals received a second infusion of $3.51 \times 10^{7}$ $1.36 \times 10^{8}$ c.f.u. $\mathrm{kg}^{-1}$ E. coli 41949 via the right jugular vein over $4-7 \mathrm{~h}$ using a syringe pump.

The animal vitals were monitored and blood samples were taken for the following analyses: blood culture, complete blood count, blood gas, blood chemistry, clotting profile and enzyme-linked lectin-sorbent assay. Organ samples were obtained at the conclusion of the study.

Bacterial organ culture. Mouse. Lung, liver, kidney and spleen tissue were collected and placed in 2-ml sterile tubes containing $2.38-\mathrm{mm}$ metal beads (MO BIO/Qiagen). Sterile saline $(1 \mathrm{ml})$ was added to each tube and the samples were weighed and then fragmented by bead-mill treatment at $30 \mathrm{~Hz}$ for $3 \mathrm{~min}$ using a Mixer Mill MM 400 machine (Verder Scientific, Inc.). The bacteria were quantified as c.f.u. $\mathrm{g}^{-1}$ from the resulting slurry by spiral plating (Eddy Jet 2, IUL), automated counting (Flash \& Go, IUL) and adjusting for the volume of water added to the organs.

Pig. To determine the bacterial load (c.f.u. $\mathrm{g}^{-1}$ ) in organs, two $0.5-1-\mathrm{cm}^{3}$ samples from four distinct locations per organ (lung, liver, kidney and spleen) were placed 
into separate pre-sterilized Nalgene bottles (Thermo Fisher Scientific) containing stainless steel balls (McMaster-Carr) and weighed. Sterile water $(17 \mathrm{ml})$ was added to each sample. The organ tissue was fragmented by bead-mill treatment at $30 \mathrm{~Hz}$ for 3 min using a Mixer Mill MM 400 machine (Verder Scientific, Inc.). The E. coli were quantified as c.f.u. $\mathrm{g}^{-1}$ from the resulting slurry by spiral plating (Eddy Jet 2, IUL), automated counting (Flash \& Go, IUL) and adjusting for the volume of water added.

ELISA for IgG antibody titres to RS218 and MRSA. For RS218, polystyrene microwell plates were functionalized (high-bind ELISA plate, 96 well; Santa Cruz Biotechnology, cat. no. sc-204463) with MBL by adding $100 \mu \mathrm{l}$ of $0.5 \mu \mathrm{g} \mathrm{ml}^{-1} \mathrm{MBL}$ (Sino Biological, Inc.) in TBST-5 $\mathrm{mM} \mathrm{CaCl}_{2}$ (Boston BioProducts). The plates were blocked overnight with $1 \%$ BSA. For MRSA, the polystyrene microwell plates were directly coated with $100 \mu \mathrm{l}$ MRSA antigens. The MRSA plates were additionally incubated in 5\% pig serum in 1\% BSA in TBST and blocked for $1 \mathrm{~h}$ and $30 \mathrm{~min}$ at room temperature. For both ELISAs, the serum samples were diluted at $1 / 100,1 / 1,000$ and $1 / 10,000$ in blocking buffer (1\% BSA in TBST) and stored at $4{ }^{\circ} \mathrm{C}$ until use. Subsequently, the wells were washed six times, and samples were added to the wells and incubated for $1 \mathrm{~h}$ and $30 \mathrm{~min}$ at room temperature. After six washes, peroxidase-AffiniPure $\mathrm{F}\left(\mathrm{ab}^{\prime}\right)_{2}$ fragment goat anti-mouse IgG (Jackson ImmunoResearch, cat. no. 111-036-047) diluted to $1 / 20,000$ was added to the wells and incubated for $1 \mathrm{~h}$ at room temperature. The wells were washed six times and $100 \mu \mathrm{l} 3,3^{\prime}, 5,5^{\prime}$-tetramethylbenzidine (TMB)-detecting HRP buffer was added. After $15 \mathrm{~min}$, the colour development was stopped with $1 \mathrm{M} \mathrm{H}_{2} \mathrm{SO}_{4}$. The optical density at $370-652 \mathrm{~nm}$ was measured using a microplate reader and the data were processed using the GraphPad Prism software. The samples were run in duplicate, unless otherwise stated, and standards were run in triplicates.

Flow cytometry. To evaluate the phenotype of cells isolated from the injection site and lymph nodes, we used panels of fluorescently conjugated monoclonal antibodies as well as additional isotype controls. Cells were stained with allophycocyanin (APC)-conjugated anti-mouse BB220 in combination with fluorescein isothiocyanate (FITC)-conjugated anti-mouse GL7, APC/ Cy7-conjugated anti-mouse CD3, BV421-conjugated anti-mouse CD4, BV711-labelled anti-mouse CD8 for detecting lymphocytes and phycoerythrin (PE)-labelled anti-mouse CD86, PE/Cy7-labelled anti-mouse CD11b, FITC-labelled anti-mouse CD11c, APC/Cy7-conjugated anti-mouse I-A/I-E and BV421-conjugated anti-mouse CD80 monoclonal antibodies. Non-specific staining was avoided by adding an FcR blocking reagent and LIVE/DEAD fixable dead cell stain. The cells were incubated for $30 \mathrm{~min}$ in the dark at $4{ }^{\circ} \mathrm{C}$ and washed in FACS buffer ( $2 \%$ fetal bovine serum in PBS) before analysis on a flow cytometer. All of the samples were analysed using a BD LSR FORTESSA flow cytometer instrument, which can detect up to 15 different fluorochrome-conjugated antibodies simultaneously. An acquisition gate was established based on forwardand side-scatter parameters that included only monocyte and lymphocyte populations and excluded dead cells and debris.

Statistical analyses. The statistical analyses in all plots were performed using $\mathrm{R}^{35}$. Statistically significant differences between multiple independent groups in Figs. 2g, 4b and Supplementary Fig. 3b were identified by implementing a non-parametric Kruskal-Wallis test. Comparisons between the naive group at day 1 and vaccine at every time point were identified by post-hoc analysis using MannWhitney $U$ test with false-discovery-rate adjustment to control for family-wise type I error. Graphs were plotted using GraphPad Prism version 8.3.0. In Figs. 3c,f, 5a, statistically significant differences between the independent groups at each time point were calculated using a non-parametric Mann-Whitney $U$ test. In Figs. 3b,e, $5 b$, statistically significant differences between the independent groups for each organ were determined using a non-parametric Mann-Whitney $U$ test. In Fig. 2d, $6 \mathrm{~b}$, statistically significant differences between the independent groups at each time point were identified using an unpaired Student's $t$-test. Results were deemed as statistically significant when the null hypothesis could be rejected with $>95 \%$ confidence. Bars represent the mean \pm s.d. in all figures.

Reporting Summary. Further information on research design is available in the Nature Research Reporting Summary linked to this article.

\section{Data availability}

The main data supporting the results in this study are available within the paper and its Supplementary Information. The proteomics data are available on the PRIDE repository under the accession code PXD023763 and can also be accessed via the MassIVE data storage at https://doi.org/10.25345/C5XB70.

Received: 12 May 2020; Accepted: 31 May 2021; Published online: 8 July 2021

\section{References}

1. Rice, L. B. Federal funding for the study of antimicrobial resistance in nosocomial pathogens: no ESKAPE. J. Infect. Dis. 197, 1079-1081 (2008).
2. Spellberg, B. et al. The epidemic of antibiotic-resistant infections: a call to action for the medical community from the Infectious Diseases Society of America. Clin. Infect. Dis. 46, 155-164 (2008).

3. Adalja, A. A. Biothreat agents and emerging infectious disease in the emergency department. Emerg. Med. Clin. North Am. 36, 823-834 (2018).

4. Messner, P., Schaffer, C. \& Kosma, P. Bacterial cell-envelope glycoconjugates. Adv. Carbohydr. Chem. Biochem. 69, 209-272 (2013).

5. Haji-Ghassemi, O., Blackler, R. J., Martin Young, N. \& Evans, S. V. Antibody recognition of carbohydrate epitopesdagger. Glycobiology 25, 920-952 (2015).

6. Nuttall, J. J. \& Eley, B. S. BCG vaccination in HIV-infected children. Tuberc. Res. Treat. 2011, 712736 (2011).

7. Wilk, M. M. et al. Immunization with whole cell but not acellular pertussis vaccines primes CD4 TRM cells that sustain protective immunity against nasal colonization with Bordetella pertussis. Emerg. Microbes Infect. 8, 169-185 (2019).

8. Liang, J. L. et al. Prevention of pertussis, tetanus, and diphtheria with vaccines in the United States: recommendations of the Advisory Committee on Immunization Practices (ACIP). MMWR Recomm. Rep. 67, 1-44 (2018).

9. Jaffe, J. et al. Effects of conformational changes in peptide-CRM197 conjugate vaccines. Bioconjug. Chem. 30, 47-53 (2019).

10. Moginger, U. et al. Cross Reactive Material 197 glycoconjugate vaccines contain privileged conjugation sites. Sci. Rep. 6, 20488 (2016).

11. Zhang, F., Lu, Y. J. \& Malley, R. Multiple antigen-presenting system (MAPS) to induce comprehensive B- and T-cell immunity. Proc. Natl Acad. Sci. USA 110, 13564-13569 (2013).

12. Kang, J. H. et al. An extracorporeal blood-cleansing device for sepsis therapy. Nat. Med. 20, 1211-1216 (2014).

13. Cartwright, M. et al. A broad-spectrum infection diagnostic that detects pathogen-associated molecular patterns (PAMPs) in whole blood. EBioMedicine 9, 217-227 (2016).

14. Seiler, B. T. et al. Broad-spectrum capture of clinical pathogens using engineered Fc-mannose-binding lectin enhanced by antibiotic treatment. F1000Res 8, 108 (2019).

15. Janeway, C. A. Jr \& Medzhitov, R. Innate immune recognition. Annu. Rev. Immunol. 20, 197-216 (2002).

16. Kim, J. et al. Injectable, spontaneously assembling, inorganic scaffolds modulate immune cells in vivo and increase vaccine efficacy. Nat. Biotechnol. 33, 64-72 (2015).

17. Ali, O. A., Huebsch, N., Cao, L., Dranoff, G. \& Mooney, D. J. Infectionmimicking materials to program dendritic cells in situ. Nat. Mater. 8, 151-158 (2009)

18. Ali, O. A. \& Mooney, D. J. Immunologically active biomaterials for cancer therapy. Curr. Top. Microbiol Immunol. 344, 279-297 (2011).

19. Wibowo, D. et al. Polymeric nanoparticle vaccines to combat emerging and pandemic threats. Biomaterials 268, 120597 (2021).

20. Ke, X. et al. Physical and chemical profiles of nanoparticles for lymphatic targeting. Adv. Drug Deliv. Rev. 151-152, 72-93 (2019).

21. Xu, W., Riikonen, J. \& Lehto, V. P. Mesoporous systems for poorly soluble drugs. Int. J. Pharm. 453, 181-197 (2013).

22. Ali, O. A. et al. Identification of immune factors regulating antitumor immunity using polymeric vaccines with multiple adjuvants. Cancer Res. 74, 1670-1681 (2014).

23. Waterhouse A., et al. Modified clinical monitoring assessment criteria for multi-organ failure during bacteremia and sepsis progression in a pig model. Advan. Crit. Care Med. 1, 002 (2018).

24. Robbins, J. B., Schneerson, R., Horwith, G., Naso, R. \& Fattom, A. Staphylococcus aureus types 5 and 8 capsular polysaccharide-protein conjugate vaccines. Am. Heart J. 147, 593-598 (2004).

25. Frenck, R. W. Jr et al. Safety and immunogenicity of a vaccine for extra-intestinal pathogenic Escherichia coli (ESTELLA): a phase 2 randomised controlled trial. Lancet Infect. Dis. 19, 631-640 (2019).

26. Shah, N. J. et al. A biomaterial-based vaccine eliciting durable tumour-specific responses against acute myeloid leukaemia. Nat. Biomed. Eng. 4, 40-51 (2020).

27. Ali, O. A., Tayalia, P., Shvartsman, D., Lewin, S. \& Mooney, D. J. Inflammatory cytokines presented from polymer matrices differentially generate and activate DCs in situ. Adv. Funct. Mater. 23, 4621-4628 (2013).

28. $\mathrm{Li}, \mathrm{A}$. W. et al. A facile approach to enhance antigen response for personalized cancer vaccination. Nat. Mater. 17, 528-534 (2018).

29. Dellacherie, M. O., Li, A. W., Lu, B. Y. \& Mooney, D. J. Covalent conjugation of peptide antigen to mesoporous silica rods to enhance cellular responses. Bioconjug. Chem. 29, 733-741 (2018).

30. Cyster, J. G. \& Schwab, S. R. Sphingosine-1-phosphate and lymphocyte egress from lymphoid organs. Annu. Rev. Immunol. 30, 69-94 (2012).

31. Lindquist, R. L. et al. Visualizing dendritic cell networks in vivo. Nat. Immunol. 5, 1243-1250 (2004).

32. Sun, L., Middleton, D. R., Wantuch, P. L., Ozdilek, A. \& Avci, F. Y. Carbohydrates as T-cell antigens with implications in health and disease. Glycobiology 26, 1029-1040 (2016).

33. Liebermeister, W. et al. Visual account of protein investment in cellular functions. Proc. Natl Acad. Sci. USA 111, 8488-8493 (2014). 
34. Sellars, B. B., Sherrod, D. R. \& Chappel-Aiken, L. Using word clouds to analyze qualitative data in clinical settings. Nurs. Manag. 49, 51-53 (2018).

35. Moran, J. L. \& Solomon, P. J. Statistics in review Part I: graphics, data summary and linear models. Crit. Care Resusc. 9, 81-90 (2007).

\section{Acknowledgements}

We thank D. Bolgen, A. Nedder, K. Imaizumi and S. Bardales for their assistance with the mouse and pig models. This work was supported by the Wyss Institute for Biologically Inspired Engineering, DARPA (grant no. W911NF-16-C-0050 to D.E.I. and M.S.) and the National Institutes of Health (grant no. 1 R01 CA223255 to D.J.M.).

\section{Author contributions}

M.S., E.J.D. and M.J.C. conceived the project, which was directed by D.E.I. and D.J.M. Vaccines were prepared and analysed by B.T.S., D.A.W., A.G.S., N.D., M.K., C.L.H.,

S.A.R., M.O.D., A.W.L. and J.M.S. Experiments in the mouse and pig models were conducted by F.L., A.R.G., K.R.L., F.R.U., C.D.Y., A.R.J. and S.L.L. The data were analysed by M.S., F.L., M.J.C., N.D. and V.C. The manuscript was written by M.S., E.J.D., D.E.I. and D.J.M. All authors critically reviewed the manuscript.

\section{Competing interests}

D.J.M. received sponsored research funding from Novartis, and has equity in Lyell and Attivare Therapeutics. D.E.I. is a founder, member of the board of directors and scientific advisory board, and equity holder in Boa Biomedical, Inc. M.S is a founder and equity holder in BOA Biomedical. E.J.D., F.L. and B.T.S. are founders and have equity in Attivare Therapeutics. Inventors, patent applications: D.J.M., D.E.I., M.S., M.J.C., E.J.D., B.T.S., F.L., A.G.S., A.R.G., J.M.S. and D.A.W. For each patent, the serial number, country and patent number are provided: (1) 15/434,781; US; 10,813,988; (2) 17/015,177; US; (3) 2018-543154; Japan; 6854530; (4) 17753811; EPO; and (5) 202000000000; China. All other authors declare that they have no competing interests.

\section{Additional information}

Supplementary information The online version contains supplementary material available at https://doi.org/10.1038/s41551-021-00756-3.

Correspondence and requests for materials should be addressed to D.J.M

Peer review information Nature Biomedical Engineering thanks Tarek Fahmy, Michael Mitchell and the other, anonymous, reviewer(s) for their contribution to the peer review of this work. Peer reviewer reports are available.

Reprints and permissions information is available at www.nature.com/reprints.

Publisher's note Springer Nature remains neutral with regard to jurisdictional claims in published maps and institutional affiliations.

(c) The Author(s), under exclusive licence to Springer Nature Limited 2021 


\section{natureresearch}

Corresponding author(s): David Mooney

Last updated by author(s): May 21, 2021

\section{Reporting Summary}

Nature Research wishes to improve the reproducibility of the work that we publish. This form provides structure for consistency and transparency in reporting. For further information on Nature Research policies, see Authors \& Referees and the Editorial Policy Checklist.

\section{Statistics}

For all statistical analyses, confirm that the following items are present in the figure legend, table legend, main text, or Methods section.

n/a Confirmed

$\bigotimes$ The exact sample size $(n)$ for each experimental group/condition, given as a discrete number and unit of measurement

$\bigotimes$ A statement on whether measurements were taken from distinct samples or whether the same sample was measured repeatedly

The statistical test(s) used AND whether they are one- or two-sided

Only common tests should be described solely by name; describe more complex techniques in the Methods section.

Х $\square$ A description of all covariates tested

$\bigotimes$ A description of any assumptions or corrections, such as tests of normality and adjustment for multiple comparisons

A full description of the statistical parameters including central tendency (e.g. means) or other basic estimates (e.g. regression coefficient)

$\triangle$ AND variation (e.g. standard deviation) or associated estimates of uncertainty (e.g. confidence intervals)

$X$ For null hypothesis testing, the test statistic (e.g. $F, t, r$ ) with confidence intervals, effect sizes, degrees of freedom and $P$ value noted

$\triangle$ Give P values as exact values whenever suitable.

Х $\square$ For Bayesian analysis, information on the choice of priors and Markov chain Monte Carlo settings

Х $\square$ For hierarchical and complex designs, identification of the appropriate level for tests and full reporting of outcomes

$\triangle \square$ Estimates of effect sizes (e.g. Cohen's $d$, Pearson's $r$ ), indicating how they were calculated

Our web collection on statistics for biologists contains articles on many of the points above.

\section{Software and code}

\section{Policy information about availability of computer code}

Data collection

No software was used.

Data analysis

R software (version 3.6.0; 2019-04-26).

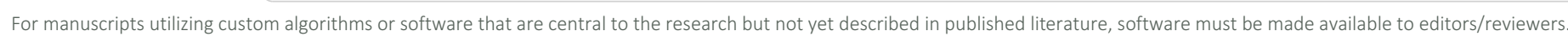
We strongly encourage code deposition in a community repository (e.g. GitHub). See the Nature Research guidelines for submitting code \& software for further information.

\section{Data}

Policy information about availability of data

All manuscripts must include a data availability statement. This statement should provide the following information, where applicable:

- Accession codes, unique identifiers, or web links for publicly available datasets

- A list of figures that have associated raw data

- A description of any restrictions on data availability

The main data supporting the results in this study are available within the paper and its Supplementary Information. The proteomics data are available on the PRIDE repository under accession code PXD023763 and can also be accessed via the MassIVE data storage at http://doi.org/10.25345/C5XB70.

\section{Field-specific reporting}

Please select the one below that is the best fit for your research. If you are not sure, read the appropriate sections before making your selection. 


\section{Life sciences study design}

All studies must disclose on these points even when the disclosure is negative.

Sample size Sample sizes were chosen on the basis of previous published reports. For the immunological assessments, we used 5 mice per group, and for the challenge experiments $10-12$ mice per group and 4 pigs per group.

Data exclusions No data were excluded from the analyses.

Replication All attempts at replication were successful.

Randomization Mice were randomized during allocation to different cages.

Blinding The animal staff allocated identification codes for each mouse. Laboratory staff were blinded to the samples. After the completion of the experiments, the codes were revealed, allowing for the compilation of clinical observation with laboratory test results.

\section{Reporting for specific materials, systems and methods}

We require information from authors about some types of materials, experimental systems and methods used in many studies. Here, indicate whether each material, system or method listed is relevant to your study. If you are not sure if a list item applies to your research, read the appropriate section before selecting a response.

Materials \& experimental systems

$\mathrm{n} / \mathrm{a}$ Involved in the study

Methods

$\square$ \ Antibodies

\ $\square$ Eukaryotic cell lines

\ Palaeontology

n/a Involved in the study

Х $\square$ ChIP-seq

$\square$ Х Flow cytometry

$\square \bowtie$ Animals and other organisms

X $\square$ MRI-based neuroimaging

Х $\square$ Human research participants

\ $\square$ Clinical data

\section{Antibodies}

Antibodies used

ELISA:Peroxidase-AffiniPure F(ab’)2 Fragment Goat anti mouse IgG (Jackson ImmunoResearch \#111-036-047.

Antibodies used for Flow Cytometry analysis:

B220 APC; Biolegend; cat \# 103212; Lot \# B242829; clone RA3-6B2

GL7 FITC; BioLegend; cat \# 144604; Lot \# B259646; clone GL7

CD4 BV421; BioLegend; cat \# 100437; Lot \# B266323; clone GK1.5

CD8 BV711; BioLegend; cat \# 100748; Lot \# B259953; clone 53-6.7

CD3 APC-Cy7; Biolegend; cat \# 100222; Lot \# B273236; clone 17a2

I-A/I-E APC-Cy7; Biolegend; cat \# 107628; Lot \# B257359; clone M5/114.15.2

CD86 PE; BioLegend; cat\# 105008; Lot \# B248266; clone GL-1

CD80 BV421; BioLegend; cat \# 104726; Lot \# B264062; clone 16-10A1

CD11b PE-Cy7; Biolegend; cat \# 101216; Lot\# B272161; clone M1/70

CD11C FITC; BioLegend; cat \# 117376; Lot \# B244374; clone N418

Validation

The HRP labeled anti mouse IgG has been used in many publications. The $F\left(a b^{\prime}\right) 2$ was used to reduce cross-reactivity. Similarly, the antibodies used in flow cytometry have been employed in many publications.

\section{Animals and other organisms}

Policy information about studies involving animals; ARRIVE guidelines recommended for reporting animal research

Laboratory animals

Wild animals

Field-collected samples

Ethics oversight
BALB/c mice, New Zealand Rabbits and Yorkshire swine.

The study did not involve wild animals.

The study did not involve samples collected from the field.

The animal studies were performed in accordance with institutional guidelines, and were approved by the Institutional Care and Use Committee of Harvard Medical School. 
Plots

Confirm that:

Х The axis labels state the marker and fluorochrome used (e.g. CD4-FITC).

Х The axis scales are clearly visible. Include numbers along axes only for bottom left plot of group (a 'group' is an analysis of identical markers).

Х All plots are contour plots with outliers or pseudocolor plots.

Х A numerical value for number of cells or percentage (with statistics) is provided.

\section{Methodology}

Sample preparation

Instrument

Software

Cell population abundance

Gating strategy
Fresh dissected solid tissues (implant site, lymph nodes and spleen) from vaccinated mice were immediately rinsed in 1x PBS to clean off any blood or other unwanted material. Mechanical dissociation of the tissues was performed to obtain a single-cell suspension. The suspension was filtered through a 70-micrometre cell strainer in order to exclude any aggregates, and immediately used for staining. All the steps were performed on ice and in sterile conditions.

\section{BD LSRFortessa}

FACS Diva 6.1 software, for data collection.

FlowJo for data analysis.

We did not do any cell sorting.

Gating strategy:

Unstained control and fluorophore-positive cells were used. Gates were drawn to collect cells expressing the specific fluorophore.

FSC-A vs. SSC-A to select lymphocytes

FSC-H vs. FSC-A to select single cells

SSC-A vs. L/D to select live cells

Additional analysis was performed to identify specific cells of interest (CD11c+MHCll+, CD3+CD4+, CD3+CD8+, BB220+).

Х Tick this box to confirm that a figure exemplifying the gating strategy is provided in the Supplementary Information. 